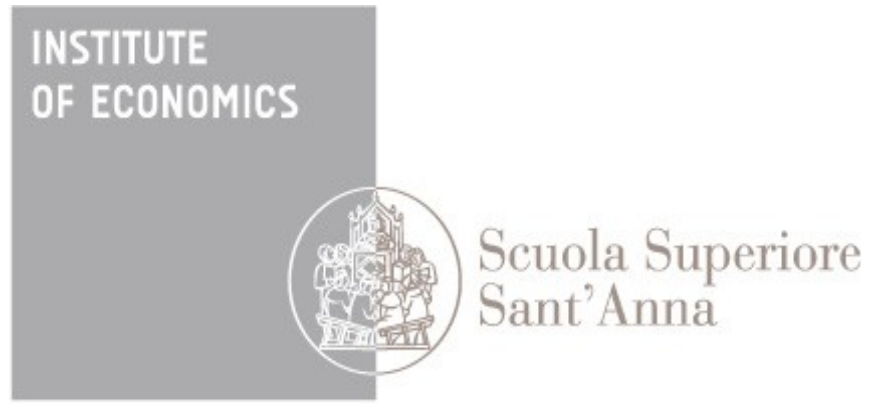

LEM | Laboratory of Economics and Management

Institute of Economics

Scuola Superiore Sant'Anna

Piazza Martiri della Libertà, 33 - 56127 Pisa, Italy ph. +3905088.33 .43$

institute.economics@sssup.it

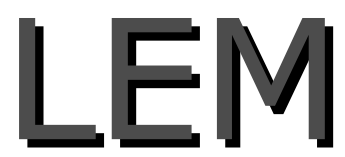

Working Paper Series

\title{
Asset prices and wealth dynamics in a financial market with endogenous liquidation risk
}

Pietro Dindo $\neq \S$

Jacopo Staccioli $\S$

\# Department of Economics, Università Ca' Foscari Venezia, Venezia, Italy

$\S$ Institute of Economics, Scuola Superiore Sant'Anna, Pisa, Italy 


\title{
Asset prices and wealth dynamics in a financial market with endogenous liquidation risk $^{*}$
}

\author{
Pietro Dindo ${ }^{\dagger}$ Jacopo Staccioli $\$$
}

18th December 2017

\begin{abstract}
Within a financial market where a risk-free bond and a long-lived risky asset are exchanged by investors with heterogeneous trading rules, we assume that the investors most exposed to the risky asset are subject to joint liquidation needs. The latter encompass a risk whenever the market impact of traders subject to them is large enough, due to a fire-sale phenomenon. Our aim is to provide conditions for the transformation of liquidation needs into liquidation risk, and to characterize the resulting asset price dynamics. We find that when the average position of traders subject to liquidation needs is lower than the position of the other traders, the former vanish and asset prices are driven solely by the dividend process. Whether liquidation risk becomes systemic or its impact is mitigated by the position of other traders, depends on the relative wealth dynamics. We provide conditions on agents positions under which the liquidation risk is always systemic because the aggressive traders dominate, as well as conditions under which the size of the liquidation risk is endogenously determined because all traders survive and the relative wealth dynamics is a mean reverting process.
\end{abstract}

JEL classification: G11, G12, C62.

Keywords: Heterogeneous Agents, Liquidation Risk, Asset Pricing, Fire-Sales, Noise Traders, Random Dynamical Systems.

\footnotetext{
*We wish to thank Mikhail Anufriev, Giulio Bottazzi, Giovanni Dosi, Fabio Tramontana, and the participants to the CEF 2016 Conference (Bordeaux, France), the SNDE 2017 Symposium (Paris, France), the WEHIA 2017 Conference (Milan, Italy), the NED 2017 Conference (Pisa, Italy), the International Workshop on Economic Growth, Macroeconomic Dynamics and Agents' Heterogeneity (St. Petersburg, Russia), and department seminars at Hokkaido University (Sapporo, Japan) and GREDEG (Valbonne, France) for helpful comments and insightful suggestions at various stages of this work. Pietro Dindo acknowledges support from the Marie Curie International Outgoing Fellowship PIOF-GA-2011-300637 within the 7th European Community Framework Programme. The usual disclaimer applies.

${ }^{+}$To whom correspondence should be addressed: $\otimes$ pietro.dindo<at>unive.it

‡Department of Economics, Università Ca' Foscari Venezia, Cannaregio 873, 30121 Venezia, Italy.

§Institute of Economics, Scuola Superiore Sant'Anna, Piazza Martiri della Libertà 33, 56127 Pisa, Italy.
} 


\section{Introduction}

In this paper, we investigate the impact of joint liquidation needs on the price dynamics of a risky asset. When a group of investors liquidate their position in a coordinated fashion, the impact on the price depends on the size of the group. If the group holds most of the asset, then a fire-sale phenomenon occurs and the asset sells at a heavily discounted price. If, otherwise, the group with joint liquidation needs is small, the market is able to absorb their supply without a significant haircut. Therefore, the transformation of joint liquidation needs into proper liquidation risk depends on the relative wealth dynamics of different groups of traders. The purpose of this paper is to study the emergence of liquidation risk by analysing the joint dynamics of risky asset prices and traders relative wealth, as triggered by liquidation needs.

In order to address this question, we consider a stylized financial economy where agents can choose between a long-lived risky security and risk-free bond. The market is populated by heterogeneous investors, each taking a position in the two assets depending on her own characteristics (e.g. risk aversion, information, beliefs, discount factor) and objective functions (e.g. payoff, utility). Investors' demand for the bond absorbs only a fraction of its total supply so that its price can be taken as fixed. Investors' demand for the risky asset instead absorbs all its supply. As a consequence the investors are exposed, other than to the (exogenous) risk of its dividend payout, also to the (endogenous) risk of possible variations in its price. If, for example, a sizeable fraction of traders (as measured by aggregate wealth) decide to sell the asset, they will be able to do so only at a lower price, as within a fire-sale. In other words, all these investors are exposed to liquidation risk, which we define as the peril of having to liquidate the asset at a substantially discounted price. On the contrary, when a small fraction of agents decides to sell the risky security, all of their demand is absorbed by the market without a significant variation in its price, making liquidation risk negligible.

Therefore, the source of liquidation risk is twofold. The first is the need to sell the risky asset in a joint coordinated fashion. When agents liquidation needs are not synchronous, the impact of each trader is negligible. Provided liquidation needs are joint, as we shall assume, the second source of risk consists of the market impact of the traders exposed to them.

In order to investigate the transformation of liquidation needs into liquidation risk, we assume that two groups of traders are active in the market. The first group is subject to joint liquidation needs, which we model as a random variable independent of the economy dynamics. There might be multiple reasons for liquidation needs to be synchronous within a group: all traders' demand is updated by looking at the same signals, e.g. an exogenous source of sentiment; alternatively, all these investors have the same institutional or financial constraints, e.g. they are all hedge funds who are overly exposed to the risky asset and might need to reduce their position due to new regulations or an industry shock. In our model the joint liquidation need is modelled as an exogenous Markov process that moves the demand of agents between two levels, either a high fraction or a low fraction of 
wealth to be invested in the risky asset. The second group of agents is not vulnerable to joint liquidation needs and has a constant exposure to the risky asset.

As it will become clear later on, the most meaningful scenario is the one in which the group that bears the liquidation hazard also acts more aggressively on the market, in the sense of committing to hold a larger share of their wealth in the risky security, relative to the other group. In this case, the liquidation needs on behalf of a single group of traders can be further rooted in their inability to cope with a systemic liquidity shortage by resorting to their shallow bond holdings to be pledged as collateral buffer.

In a dynamic setting, where taking aggressive position carries liquidation risk, a tradeoff between different sources of risk clearly emerges. On the one hand, taking more fundamental risk brings a positive reward since riskier securities carry a higher expected return (when looking only at the exogenous component of risk). On the other hand, a portfolio which is exceedingly skewed towards the risky security also turns out to be vulnerable to losses due to the said fire-sale phenomenon. When all aggressive traders sell and their market impact is high, they have to bear a substantial haircut, which we identify as liquidation risk. We are able to analytically investigate this trade-off and provide sufficient conditions for the emergence of endogenous liquidation risk in equilibrium.

From a mathematical point of view, the model outlined above can be described by a random dynamical system. The state variables are the agents' relative wealth, the risky asset's return, and the dividend yield. The random shocks play the role of the joint liquidation needs. Contrary to the incumbent literature of heterogeneous agent model (reviewed in the following Section), such a system cannot be studied simply by perturbing its deterministic skeleton. Since the joint liquidation needs constitute the drivers of the dynamics, their effect cannot be switched off. Technically, we study the local stability of the underlying deterministic and stochastic steady states. We shall provide sufficient conditions under which either the traders with a constant exposure dominate, i.e. there is no liquidation risk, or the traders subject to liquidation risk dominate, i.e. there is systemic liquidation risk in equilibrium. The most interesting scenario from an economic standpoint arises when both these polar equilibria are simultaneously unstable. In this case, the extent of the liquidation risk becomes endogenous and is coupled with the agents' relative wealth dynamics.

Our results are as follows. We find that a riskier portfolio may systematically fail to outperform a relatively safer one, should the former incorporate the aforementioned liquidation shocks. The emergence of endogenous liquidation risk, due to long-run heterogeneity of traders either subject or immune to the joint liquidation needs, arises as a generic outcome, i.e. it occurs for a non-degenerate region of the parameters space. Somewhat counter-intuitively, both trader types may co-exist even when the constant trader always invests a strictly lower share of her wealth in the risky security, compared to the stochastic trader. Losses due to fire-sales can be, on average, so severe that both groups of traders survive. Moreover, we find that the constant portfolio can systematically earn a higher return and eventually dominate the economy, even if it consists of a position which is on average strictly less than that of the stochastic one. Overall, the transformation of liquidation needs into liquidation risk brings an intrinsic penalty to the stochastic trader due 
to the well known effect of buying high and selling low associated with her fire-sale-like behaviour.

The paper is organised as follows. The next Section reviews the related literature. In Section 2 the financial model is outlined and the underlying random dynamical system governing the economy is derived. Then, Section 3 analyses the representative agent restriction, namely the no liquidation risk case and the systemic liquidation risk case. These two extreme cases are useful for the analysis of the full model, presented in Section 4. Here, sufficient conditions for all possible long-run outcomes and the related emergence of endogenous liquidation risk are obtained. Section 5 provides numerical simulation evidence of some interesting scenarios generated by our model and the sensitivity analysis for its relevant parameters. Finally, Section 6 concludes and proposes conceivable extensions to our framework.

\subsection{Related literature}

By assuming that the market is populated by two classes of traders, our model is akin to the still flourishing literature of Heterogeneous Agent Models (hereafter HAMs); see e.g. Hommes (2006) for a survey. The literature has emerged with the aim of modelling possible sources of endogenous risk. In particular, models close to ours that investigate the joint asset prices and wealth dynamics are Chiarella and He (2001), Chiarella et al. (2006), Anufriev et al. (2006), Anufriev and Bottazzi (2010), Anufriev and Dindo (2010), Evstigneev et al. (2011), and Anufriev et al. (2012). However, to the best of our knowledge, no contribution has so far analysed the transformation of random liquidation needs in liquidation risk and its impact on the asset price dynamics.

Assuming joint liquidation needs that are modelled as an exogenous stochastic process, our contribution is also linked to the concept of noise traders and to the impact of noise unrelated to the exogenous dividend process, as put forth by Black (1986). In fact, assuming that the liquidation need is a random variable implies that a group of traders' demand is stochastic. The concept has been incorporated by different streams of literature to include portfolio decisions that are not strictly based on rational expectations. However, the HAMs literature has traditionally modelled noise traders with strategies driven by deterministic feedback mechanisms from realised market outcomes, mainly in terms of chartist (i.e. trend extrapolating) versus fundamentalist (i.e. mean reverting) rules (see e.g. Chiarella, 1992, Brock and Hommes, 1998).

Little has been done so far to study the interaction between strategies incorporating a truly random component that continually perturbs the system away from equilibrium. In fact, it is not infrequent to spot well-defined stochastic processes and other sources of noise in many HAMs. However, the effect of such randomness on the long-run dynamics of the system is often deliberately nullified by the adoption, for tractability purposes, of the so-called deterministic skeleton approach: when this is used, any realisation of a random variable is substituted by its expected value, and any higher statistical moment is essentially discarded. Although it is widely recognised that this, so-called indirect, approach might fail to properly characterise the dynamics of the underlying stochastic system, very 
few studies have addressed the issue of exactly identifying the loss of generality induced by adopting this practice. A notable exception is Chiarella et al. (2011), which employs a direct and analytic approach to study a traditional fundamentalists vs. chartists asset pricing model, in which the fundamental price follows a random walk process. The authors find that when the memory of the fundamentalist traders approaches zero, the stochastic dynamics displays very different features from those of the underlying deterministic skeleton.

Most of our results depend on the fact that when a liquidation shock hits those traders who hold most of the risky asset supply, a fire-sale kicks in as in Shleifer and Vishny (2011). This phenomenon is especially exacerbated when the related assets are somewhat 'specialised' and the shock is industry-wide. In this case, high potential bidders from the same industry are themselves in financial shortage and more likely to sell, rather than purchase, similar assets. The assets are eventually bought at a large discount by non-specialist outsiders, distinguished by a much lower valuation than industry peers. ${ }^{1}$ The consequences of a liquidity crisis can easily turn out pathological. On a theoretical ground, Caballero and Simsek (2013) provide a model of self-reinforcing propagation of financial distress stemming from a domino effect of fire-sales fostered by endogenously increasing uncertainty in the banking sector. Studying mutual fund transactions between 1980 and 2004, Coval and Stafford (2007) show that even in the most liquid equity markets there can be a significant premium for immediacy, and price effects are relatively long-lasting. The tight connection between funding and market liquidity is again apparent, and seems to apply even when the initial 'disorder' is far from being economy-wide. Within this context, Wagner (2011) studies the optimal portfolio choice, taking into account this systemic liquidation risk.

\section{The model}

Our framework is common to a number of contributions in the HAMs literature, in particular Anufriev et al. (2006) and Anufriev and Dindo (2010). We first lay down the general market model and then introduce our specific assumptions regarding traders' behaviour in Section 2.1.

Consider a financial economy where a long-lived risky stock and a risk-free bond are traded in discrete time $t \in \mathbb{N} \cup\{0\}$. The amount of circulating shares of the risky security is constant, while the supply of the bond is perfectly elastic. The market is populated by an arbitrarily large number of traders divided in two groups indexed by $i=1,2$. The detailed behaviour of each group is described shortly afterwards.

Before trade at time $t$ starts, each trader in group $i$ chooses the fraction $x_{i, t}$ of her current wealth $W_{i, t}$ to be invested in the risky asset. The latter pays a non-negative dividend $D_{t}$ at the beginning of each period. The residual fraction of wealth, $1-x_{i, t}$, is allocated to bond purchase, yielding a constant risk-free rate of return $r_{f}>0$. The current level of individual wealth for group $i$ depends on the past trading decision at time $t-1$, i.e. on the relative allocation of wealth between the risky and the risk-free investment, on the

\footnotetext{
${ }^{1}$ See e.g. Pulvino (1998) for an insightful empirical study on the market for commercial aircraft.
} 
amount of dividend $D_{t}$ that is paid, and on security prices. The price of the risky asset $P_{t}$ is determined in equilibrium by equating aggregate demand and aggregate supply. Both the price and the dividend are expressed in terms of the bond's price, serving as the numéraire, conventionally normalised to 1 in every period. The evolution of wealth for the agents within group $i$ holds

$$
W_{i, t}=\left(1-x_{i, t-1}\right) W_{i, t-1}\left(1+r_{f}\right)+x_{i, t-1} W_{i, t-1}\left(\frac{P_{t}+D_{t}}{P_{t-1}}\right) .
$$

The economy runs through a series of temporary equilibria in which the market clearing condition is satisfied. By normalising, without loss of generality, the supply of the risky security to 1 , market clearing condition amounts to

$$
P_{t}=\sum_{i=1,2} x_{i, t} W_{i, t}
$$

The system of Eqs. (2.1) and (2.2) describes a growing economy at a rate that is in part deterministic, corresponding to the risk-free return $r_{f}$, and in part dependent on the realised price and dividend. It is easy to check this by summing the individual wealth over all the agents

$$
W_{t}=\sum_{i=1,2} W_{i, t}=W_{t-1}\left(1+r_{f}\right)+\left[P_{t}+D_{t}-P_{t-1}\left(1+r_{f}\right)\right] .
$$

It is therefore useful to get rid of the constant growth component $r_{f}$ and define the rescaled variables

$$
w_{i, t}=\frac{W_{i, t}}{\left(1+r_{f}\right)^{t}}, \quad p_{t}=\frac{P_{t}}{\left(1+r_{f}\right)^{t}}, \quad d_{t}=\frac{D_{t}}{\left(1+r_{f}\right)^{t}},
$$

and the associated rescaled model

$$
\left\{\begin{array}{l}
p_{t}=\sum_{i=1,2} x_{i, t} w_{i, t} \\
w_{i, t}=w_{i, t-1}\left[1+x_{n, t-1}\left(\frac{p_{t}}{p_{t-1}}-1+e_{t}\right)\right]
\end{array}\right.
$$

where $e_{t}$ stands for the (rescaled) dividend yield, defined as

$$
e_{t}:=\frac{D_{t}}{P_{t-1}\left(1+r_{f}\right)}=\frac{d_{t}}{p_{t-1}} .
$$

Note that in the first equation of system (2.5) the current price level $p_{t}$ appears both in the LHS and in the RHS, as determinant of the level of wealth $w_{i, t}$. Substituting the second equation into the first and solving for $p_{t}$ yields the explicit evolution of the price

$$
p_{t}=p_{t-1} \frac{\sum_{i=1,2} w_{i, t-1} x_{i, t}\left[1+x_{i, t-1}\left(e_{t}-1\right)\right]}{\sum_{i=1,2} w_{i, t-1} x_{i, t-1}\left(1-x_{i, t}\right)} .
$$


It will prove useful for the subsequent analysis to normalise each group's wealth by the total wealth. Computing the wealth share $\varphi_{i, t}$ we obtain

$$
\varphi_{i, t}:=\frac{w_{i, t}}{\sum_{i=1,2} w_{i, t}}=\varphi_{i, t-1} \frac{1+x_{i, t-1}\left(r_{t}+e_{t}\right)}{1+\left(r_{t}+e_{t}\right) \sum_{i=1,2} \varphi_{i, t-1} x_{i, t-1}},
$$

where the last equality comes from the second equation of system (2.5), and $r_{t}$ denotes the capital gain (in excess of the risk-free return). It is immediate to compute the latter in terms of individual wealth shares from Eqs. (2.7) and (2.8) as follows:

$$
r_{t}:=\frac{p_{t}}{p_{t-1}}-1=\frac{\sum_{i=1,2} \varphi_{i, t-1}\left[x_{i, t}\left(1+e_{t} x_{i, t-1}\right)-x_{i, t-1}\right]}{\sum_{i=1,2} \varphi_{i, t-1} x_{i, t-1}\left(1-x_{i, t}\right)} .
$$

The return depends on the wealth-weighted average of the relative portfolio position of each group between the current and the preceding period, and on the dividend yield process. Regarding the latter, in the past literature a few distinct practices emerge. Anufriev and Bottazzi (2010), Anufriev et al. (2006) and Chiarella and He (2001) all assume an endogenous dividend dynamics such that the dividend yield is an i.i.d. process; this directly implies that any change in the price of the risky security causes an essentially instantaneous adjustment in the paid dividend of an identical magnitude. Evstigneev et al. (2011) instead anchor the dividend to the aggregate wealth in the economy. As opposed to linking the dividend to the endogenous dynamics of the economy, Chiarella et al. (2006) and Anufriev and Dindo (2010) implement an exogenously growing dividend process with i.i.d. rate of growth. We follow the latter proposal but for the sake of simplicity, in view of the already cumbersome effect of random liquidation needs, we opt for a fully deterministic process. Switching the dividend noise off allows us to focus on traders' behaviour as the only source of randomness in the model, and therefore neglect the effect of the arguably non-trivial ${ }^{2}$ correlation structure between the two processes. That said, we make the following assumption:

Assumption 1. The paid dividend grows geometrically at a rate $g>0$

$$
d_{t}=d_{t-1}(1+g) \text {. }
$$

We deliberately restrict to a (strictly) positive rate of growth since our focus is on the selective capacity of markets where risky asset, in the absence of liquidation risk, yield a higher coupon with respect to the risk-free security (at least on average). Strictly speaking, our Assumption clearly introduces profit opportunities whenever the stock price grows at a constant rate, since $g>0$ implies that the non-rescaled dividend $D_{t}$ grows at a greater pace than the risk-free return $r_{f}$. However, we shall see that the presence of endogenous liquidation risk might wipe such profit opportunities away.

\footnotetext{
${ }^{2}$ An extension of the present model featuring a stochastic dynamics of the dividend could be an interest issue to be addressed in future research.
} 
In terms of the dividend yield, Assumption 1 translates into

$$
e_{t}=\frac{d_{t}}{p_{t-1}}=e_{t-1} \frac{1+g}{1+r_{t-1}} .
$$

It is apparent in Eq. (2.11) that a negative feedback coming from the past realised return negatively affects the current level of the yield. Eqs. (2.8), (2.9), and (2.11) together describe the overall dynamics of the wealth shares, the rate of return, and the dividend yield. They can be summarised in the following 3-dimensional system:

$$
\left\{\begin{array}{l}
\varphi_{1, t}=\varphi_{1, t-1} \frac{1+x_{1, t-1}\left(r_{t}+e_{t}\right)}{1+\left(r_{t}+e_{t}\right) \sum_{i=1,2} \varphi_{1, t-1} x_{1, t-1}} \\
r_{t}=\frac{\sum_{i=1,2} \varphi_{i, t-1}\left[x_{i, t}\left(1+e_{t} x_{i, t-1}\right)-x_{i, t-1}\right]}{\sum_{i=1,2} \varphi_{i, t-1} x_{i, t-1}\left(1-x_{i, t}\right)} \\
e_{t}=e_{t-1} \frac{1+g}{1+r_{t-1}}
\end{array} .\right.
$$

Notice that since, by definition, $\sum_{i=1,2} \varphi_{i, t}=1$, the fraction of wealth of the second group can be residually derived as $\varphi_{2, t}=1-\varphi_{1, t}$.

\subsection{Trader behaviour}

The specification of traders' investment choice $x_{i, t}, i=1,2$, closes the model. For tractability purposes we restrict investment rules to those of a fixed-mix type (see Mulvey and Kim, 2010). As long as a specific fixed-mix rule is adopted, each trader rebalances her portfolio to keep the weight $x_{i}$ of the risky security unchanged by selling (respectively, buying) the asset if its price has increased (respectively, decreased).

In order to be economically meaningful, the price of an asset supplied in finite amount needs to be, at the very least, strictly positive. A sufficient condition thereof, stemming from Eq. (2.7) and widely adopted in the literature, coincides with preventing each trader from short-selling or leverage-buy the risky asset, i.e. requiring $x_{i, t} \in(0,1), i=1,2$. Note that this condition is not necessary, since the restriction only needs to apply to the aggregate market-portfolio. Note also that this assumption does not stand at odds with empirical evidence: for instance, studying a sample of U.S. domestic equity funds in the 1994-2000 period, Almazan et al. (2004) show that short-sale restrictions are not exceptional, due to both regulatory and self-imposed constraints.

The first group of traders is characterised by a constant $x_{1, t}=\bar{x}$. We shall name this group the constant group and denote it by $\mathfrak{C}$. The second group of traders enact a likewise constant exposure to the risky asset, but are subject to random liquidation shocks. In normal times these traders adopt the rule $x_{2, t}=x^{u}$. When a liquidation shock hits, say at time $\bar{t}$, the exposure to the risky asset decreases to $x_{2, \bar{t}}=x^{d}<x^{u}$. We also assume that the exposure rests at the lower level $x^{d}$ for a prolonged amount of time, before recovering to the higher level $x^{u}$. Hence, the portfolio of traders in such group consists of a stochastic variable with two admitted values. In particular, we model the transition between these two 


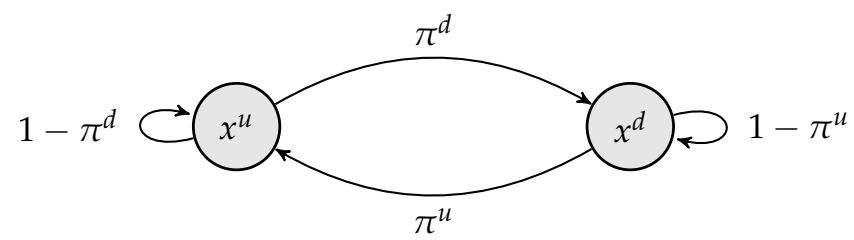

Figure 1: The Markov process $\left\{x_{t}, t \in \mathbb{N}\right\}$.

levels by an exogenous Markov process. We shall name this group the stochastic group and denote it by $\mathfrak{S}$.

The following Assumption fully summarises the behaviour of the two groups of agents.

Assumption 2. Each trader in group $\mathbb{C}$ adopts the portfolio rule $\bar{x} \in(0,1)$ in every period.

Each trader in group $\mathfrak{S}$ adopts the portfolio rule $x_{t} \in\left\{x^{u}, x^{d}\right\}$ according to a Markov process $\left\{x_{t}, t \in \mathbb{N}\right\}$, characterised by the following transition probability matrix:

$$
\mathcal{P}={ }_{x^{d}}^{x^{u}}\left(\begin{array}{cc}
x^{u} & x^{d} \\
1-\pi_{d} & \pi^{d} \\
\pi^{u} & 1-\pi^{u}
\end{array}\right),
$$

where

$$
0<x^{d}<x^{u}<1, \quad \pi^{u}>0, \quad \pi^{d}>0 .
$$

Given the transition probabilities $\mathcal{P}$, we name $\mathbb{P}$ the induced probability measure on the probability space created by the sequences $\left\{x_{t}, t \in \mathbb{N}\right\} \in X_{\infty}\left\{x^{u}, x^{d}\right\}$.

The Markov process $\left\{x_{t}\right\}$, also pictured in Fig. 1, constitutes the driver of liquidation shocks, both for what concerns their occurrence and the time needed to recover from them. The transition probability $\pi^{d}$ determines the probability of having a liquidation shock, conditional on being exposed to the risky asset at a 'up' level. Liquidation shocks only occur when the exposure to the risky asset is high. The transition probability $\pi^{u}$ determines the probability of recovering from a liquidation shock (i.e. from the 'down' level). Given the transition probabilities, the average duration of a 'down' or a ' $u p^{\prime}$ ' level of investment on a given realisation $\left\{x_{t}\right\}_{t=0}^{\infty}$ equals $\left(\pi^{d}\right)^{-1}$ and $\left(\pi^{u}\right)^{-1}$, respectively.

In terms of the two groups just outlined, the 3-dimensional random dynamical system is the composition of group $\mathfrak{S}$ stochastic demand $\left\{x_{t}\right\}$ and of the four maps $\mathcal{F}_{x_{t-1}, x_{t}}: \mathcal{D} \rightarrow \mathcal{D}$, 
each defined over the phase space $\mathcal{D}=\Delta \times(-1,+\infty) \times \mathbb{R}_{++}$and given by

$$
\mathcal{F}_{x_{t-1}, x_{t}}:\left\{\begin{array}{l}
\varphi_{t}=\varphi_{t-1} \frac{1+x_{t-1}\left(r_{t}+e_{t}\right)}{1+\left(r_{t}+e_{t}\right)\left[\varphi_{t-1} x_{t-1}+\left(1-\varphi_{t-1}\right) \bar{x}\right]} \\
r_{t}=\frac{\varphi_{t-1}\left[x_{t}\left(1+e_{t} x_{t-1}\right)-x_{t-1}\right]+\left(1-\varphi_{t-1}\right) e_{t} \bar{x}^{2}}{\varphi_{t-1} x_{t-1}\left(1-x_{t}\right)+\left(1-\varphi_{t-1}\right) \bar{x}(1-\bar{x})} \\
e_{t}=e_{t-1} \frac{1+g}{1+r_{t-1}}
\end{array}\right.
$$

where we adopt the convention that $\varphi_{t}$ denotes the time- $t$ aggregate wealth share of traders within group $\mathfrak{S}$, i.e.

$$
\varphi_{t}:=\frac{w_{\mathfrak{S}, t}}{w_{\mathfrak{C}, t}+w_{\mathfrak{S}, t}}
$$

so that $1-\varphi_{t}$ residually stands for the time-t aggregate wealth share of traders within group $\mathbb{C}$.

\section{Representative trader economies}

Let us first consider an economy populated only by traders of the same type, either constant or stochastic. This case is insightful since it allows to properly disentangle the features of the long-run dynamics implied by each behavioural specification from those stemming from the market interaction induced by liquidation shocks. The resulting reduced system is lower dimensional with respect to (2.15) since the underlying wealth share $\varphi_{t}$ is fixed to zero (respectively, one) whenever we consider an economy populated uniquely by constant (respectively, stochastic) traders.

\subsection{The economy with a constant trader: no liquidation risk}

The reduced system $\tilde{\mathcal{F}}^{\mathbb{C}}: \tilde{\mathcal{D}} \rightarrow \tilde{\mathcal{D}}$, over the phase space $\tilde{\mathcal{D}}=(-1,+\infty) \times \mathbb{R}_{++}$is fully deterministic and reads

$$
\tilde{\mathcal{F}}^{\mathbb{C}}:\left\{\begin{array}{l}
r_{t}=e_{t} \frac{\bar{x}}{1-\bar{x}} \\
e_{t}=e_{t-1} \frac{1+g}{1+r_{t-1}}
\end{array}\right.
$$

By lagging and substituting the first equation into the second, it is easy to further reduce system (3.1) to a 1-dimensional map $\tilde{f}^{\mathfrak{C}}: \mathbb{R}_{++} \rightarrow \mathbb{R}_{++}$solely in terms of the dividend yield:

$$
e_{t}=\tilde{f}^{\mathfrak{C}}\left(e_{t-1}\right)=e_{t-1} \frac{1-\bar{x}}{1+\bar{x}\left(e_{t-1}-1\right)}(1+g) .
$$

Definition 1. A steady state of system (3.1) is a vector $\left(r^{*}, e^{*}\right) \in \tilde{\mathcal{D}}$ such that $\left(r^{*}, e^{*}\right)=$ $\tilde{\mathcal{F}}^{\mathbb{C}}\left(r^{*}, e^{*}\right)$. 
The following Proposition characterises the asymptotic dynamics of the economy.

Proposition 1. The market dynamics (3.1) admits a unique, globally stable, steady state

$$
\tilde{\mathbb{C}}=\left(g, g \frac{1-\bar{x}}{\bar{x}}\right) .
$$

Proof. See Appendix A.

The price of the risky security grows, proportionally to the dividend, at a rate of $g$. Intuitively, since the asset is in finite constant supply, its price has to fully account for the new wealth injected by means of the paid dividend. From the second equation of system (2.5), since the portfolio rule $\bar{x}$ is itself constant, the market clearing price must adjust accordingly.

\subsection{The economy with a stochastic trader: systemic liquidation risk}

For each pair $\left(x_{t-1}, x_{t}\right) \in \mathrm{X}_{2}\left\{x^{u}, x^{d}\right\}$, when all traders are affected by a synchronous liquidity shock, the returns and dividend yield dynamics $\tilde{\mathcal{F}}_{x_{t-1}, x_{t}}^{\mathfrak{S}}: \tilde{\mathcal{D}} \rightarrow \tilde{\mathcal{D}}$ over the phase space $\tilde{\mathcal{D}}=(-1,+\infty) \times \mathbb{R}_{++}$read

$$
\tilde{\mathcal{F}}_{x_{t-1}, x_{t}}^{\mathcal{S}}:\left\{\begin{array}{l}
r_{t}=\frac{x_{t}\left(1+e_{t} x_{t-1}\right)-x_{t-1}}{x_{t-1}\left(1-x_{t}\right)} \\
e_{t}=e_{t-1} \frac{1+g}{1+r_{t-1}}
\end{array} .\right.
$$

By lagging and substituting the first equation into the second, it is easy to further reduce system (3.4) to a 1-dimensional stochastic map $\tilde{f}_{x_{t-2}, x_{t-1}}^{\mathfrak{S}}: \mathbb{R}_{++} \rightarrow \mathbb{R}_{++}$solely in terms of the dividend yield:

$$
e_{t}=\tilde{f}_{x_{t-2}, x_{t-1}}^{\mathfrak{S}}\left(e_{t-1}\right)=e_{t-1} \frac{x_{t-2}\left(1-x_{t-1}\right)}{x_{t-1}\left[1+x_{t-2}\left(e_{t-1}-1\right)\right]}(1+g) .
$$

Notice that this map, also pictured ${ }^{3}$ in Fig. 2(a), only depends on the past realisations $x_{t-1}$ and $x_{t-2}$ of the Markov process $\left\{x_{t}\right\}$, while the current realisation $x_{t}$ plays no role in determining the current level of the dividend yield $e_{t}$. As a matter of notation, we shall use $x_{-1}$ and $x_{-2}$ to denote the 1-period and 2-period lagged values of the generic realization of the shock $x$.

Definition 2. Call $\tilde{\mathcal{D}}^{*}$ the space of all random vectors $\left(R^{*}, E^{*}\right): X_{2}\left\{x^{u}, x^{d}\right\} \rightarrow \tilde{\mathcal{D}}$. A steady state of system (3.4) is a random vector $\left(R^{*}, E^{*}\right) \in \tilde{\mathcal{D}}^{*}$ such that $\left(R^{*}, E^{*}\right)_{x_{-1}, x}=\tilde{\mathcal{F}}_{x_{-1}, x}\left(R^{*}, E^{*}\right)_{x_{-2}, x_{-1}}$, for all $\left(x_{-2}, x_{-1}\right)$ and $\left(x_{-1}, x\right)$ in $X_{2}\left\{x^{u}, x^{d}\right\}$.

The following Proposition shows that, in an economy where all traders are subject to liquidation shocks, there exists a globally stable stochastic steady state.

\footnotetext{
${ }^{3}$ Fig. 2(a) is intended as a qualitative picture; it is not generally true that $\lim _{e_{-1} \rightarrow 0^{+}} \tilde{f}_{d u}^{\prime}>1$ since the slope of the map in fact depends on the underlying value of $g$.
} 


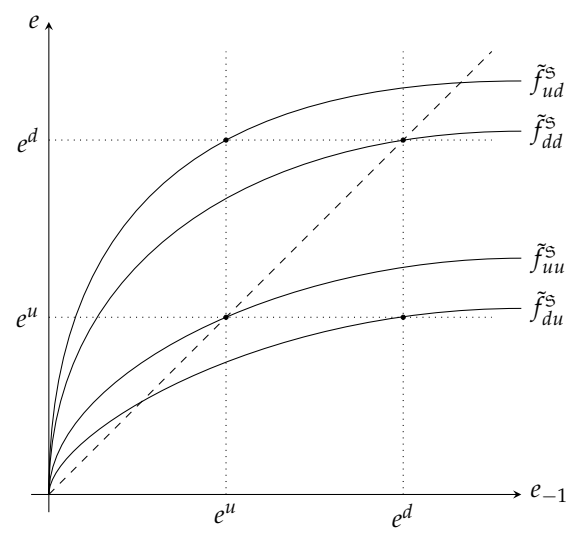

(a)

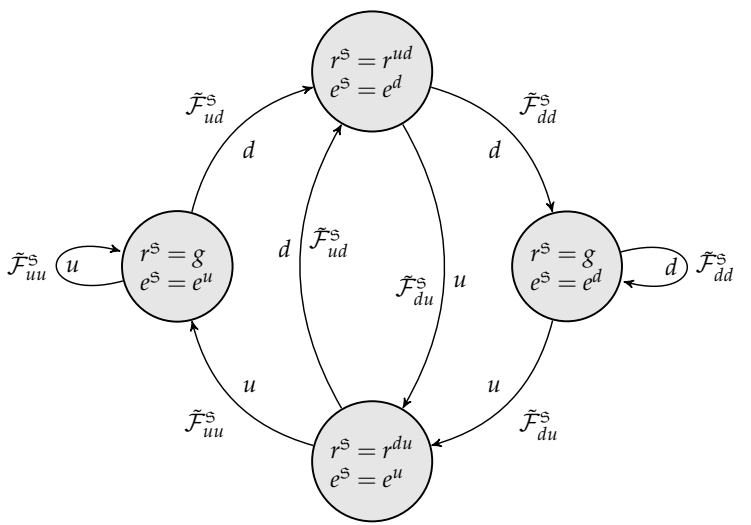

(b)

Figure 2: (a) The four realisations of the stochastic map $\tilde{f}_{x_{t-2}, x_{t-1}}$ and the bisector line (dashed). (b): The stochastic 4-cycle associated with steady state $\tilde{\mathfrak{S}}$.

Proposition 2. For every realization of the Markov process $\left\{x_{t}\right\}$, the market dynamics (3.4) admits a unique, steady state

$$
\tilde{\mathfrak{S}}=\left(g \frac{x\left(1-x_{-1}\right)}{x_{-1}(1-x)}+\frac{x-x_{-1}}{x_{-1}(1-x)}, g \frac{1-x_{-1}}{x_{-1}}\right), \quad \forall\left(x_{-1}, x\right) \in \chi_{2}\left\{x^{u}, x^{d}\right\} .
$$

Moreover, $\tilde{\mathfrak{S}}$ is globally stable:

$$
\lim _{t \rightarrow \infty} \tilde{\mathcal{F}}_{x_{-1}, x}^{\mathcal{S}}\left(\tilde{\mathcal{F}}_{x_{t}, x_{-1}}^{\mathfrak{S}} \circ \tilde{\mathcal{F}}_{x_{t-1}, x_{t}}^{\mathfrak{S}} \circ \cdots \circ \tilde{\mathcal{F}}_{x_{0}, x_{1}}^{\mathfrak{S}}\left(R_{0}, E_{0}\right)\right)=\left(R^{*}, E^{*}\right)_{x_{-1}, x}
$$

for every sequence $\left\{x_{t}\right\}$, for all $\left(x_{-1}, x\right) \in X_{2}\left\{x^{u}, x^{d}\right\}$, and for every initial condition $\left(R_{0}, E_{0}\right) \in$ $\tilde{\mathcal{D}}$.

Proof. See Appendix B.

Corollary 1. The steady state $\tilde{\mathfrak{S}}$ is an irreducible, time-homogeneous Markov chain characterised by the following transition probability matrix

$$
\left.\tilde{\mathcal{P}}=\begin{array}{c}
\left(x^{u}, x^{u}\right) \\
\left(x^{u}, x^{d}\right) \\
\left(x^{u}, x^{u}\right) \\
\left(x^{d}, x^{d}\right)
\end{array}\right)\left(\begin{array}{cccc}
1-\pi^{d} & \left.x^{u}, x^{d}\right) & \left(x^{d}, x^{u}\right) & \left(x^{d}, x^{d}\right) \\
0 & 0 & 1-\pi^{u} & \pi^{u} \\
1-\pi^{d} & \pi^{d} & 0 & 0 \\
0 & 0 & 1-\pi^{u} & \pi^{u}
\end{array}\right) .
$$

Since all states are positive-recurrent, there exists a unique invariant distribution $\tilde{\pi}$ satisfying the 
condition $\tilde{\boldsymbol{\pi}}=\tilde{\boldsymbol{\pi}} \tilde{\mathcal{P}}$. In particular it holds

$$
\tilde{\boldsymbol{\pi}}=\left[\frac{\pi^{u}\left(1-\pi^{d}\right)}{\pi^{u}+\pi^{d}}, \frac{\pi^{u} \pi^{d}}{\pi^{u}+\pi^{d}}, \frac{\pi^{u} \pi^{d}}{\pi^{u}+\pi^{d}}, \frac{\pi^{d}\left(1-\pi^{u}\right)}{\pi^{u}+\pi^{d}}\right] .
$$

The steady state $\tilde{\mathfrak{S}}$ consists of 4 'periodic' states $\left(R^{*}, E^{*}\right)_{x_{-2}, x_{-1}}^{\tilde{\mathfrak{S}}} \in \tilde{\mathcal{D}}$, associated to all possible couples $\left(x_{-2}, x_{-1}\right) \in X_{2}\left\{x^{u}, x^{d}\right\}$. The four maps $\tilde{\mathcal{F}}_{x_{-1}, x}^{\mathcal{S}}$, associated to all possible couples $\left(x_{-1}, x\right) \in X_{2}\left\{x^{u}, x^{d}\right\}$, govern the transition between them, as depicted in Fig. 2(b). In particular, there exist:

$\diamond$ three equilibrium values of the return

$$
r^{\tilde{\mathfrak{s}}}\left(x_{-1}, x\right)=\left\{\begin{array}{lll}
g & \text { if } \quad x_{-1}=x \\
r^{u d}=g \frac{x^{d}\left(1-x^{u}\right)}{x^{u}\left(1-x^{d}\right)}-\frac{x^{u}-x^{d}}{x^{u}\left(1-x^{d}\right)} & \text { if } \quad\left(x_{-1}, x\right)=\left(x^{u}, x^{d}\right), \\
r^{d u}=g \frac{x^{u}\left(1-x^{d}\right)}{x^{d}\left(1-x^{u}\right)}+\frac{x^{u}-x^{d}}{x^{d}\left(1-x^{u}\right)} & \text { if } \quad\left(x_{-1}, x\right)=\left(x^{d}, x^{u}\right)
\end{array}\right.
$$

$\diamond$ two equilibrium values of the dividend yield

$$
e^{\tilde{\mathfrak{S}}}\left(x_{-1}, x\right)=\left\{\begin{array}{lll}
e^{u}=g \frac{1-x^{u}}{x^{u}} & \text { if } & x_{-1}=x^{u} \\
e^{d}=g \frac{1-x^{d}}{x^{d}} & \text { if } & x_{-1}=x^{d}
\end{array},\right.
$$

$\diamond$ and four maps

$$
\tilde{\mathcal{F}}_{x_{-1}, x}^{\mathfrak{S}}\left(R^{*}, E^{*}\right)_{x_{-2}, x_{-1}}:\left\{\begin{array}{l}
\frac{x\left[1+e^{\tilde{S}}\left(x_{-1}, x\right)\right]-x_{-1}}{x_{-1}(1-x)} \\
e^{\tilde{S}}\left(x_{-2}, x_{-1}\right) \frac{1+g}{1+r^{\tilde{S}}\left(x_{-2}, x_{-1}\right)}
\end{array} \quad \forall x_{-2}, x_{-1}, x \in\left\{x^{u}, x^{d}\right\}\right.
$$

such that the 'periodic' states read

$$
\begin{array}{ll}
\left(R^{*}, E^{*}\right)_{u u}^{\tilde{\mathfrak{S}}}:\left\{\begin{array}{l}
r^{\tilde{\mathfrak{S}}}=g \\
e^{\tilde{\mathfrak{S}}}=e^{u}
\end{array}, \quad\left(R^{*}, E^{*}\right)_{d d}^{\tilde{\mathfrak{S}}}:\left\{\begin{array}{l}
r^{\tilde{\mathfrak{S}}}=g \\
e^{\tilde{\mathfrak{S}}}=e^{d}
\end{array},\right.\right. \\
\left(R^{*}, E^{*}\right)_{u d}^{\tilde{\mathfrak{S}}}:\left\{\begin{array}{l}
r^{\tilde{\mathfrak{S}}}=r^{u d} \\
e^{\tilde{\mathfrak{S}}}=e^{u}
\end{array}, \quad\left(R^{*}, E^{*}\right)_{d u}^{\tilde{\mathfrak{S}}}:\left\{\begin{array}{l}
r^{\tilde{\mathfrak{S}}}=r^{d u} \\
e^{\tilde{\mathfrak{S}}}=e^{d}
\end{array},\right.\right.
\end{array}
$$


and the following equalities are satisfied

$$
\begin{aligned}
& \left(R^{*}, E^{*}\right)_{u u}^{\tilde{\mathcal{S}}}=\tilde{\mathcal{F}}_{u u}^{\mathcal{S}}\left(R^{*}, E^{*}\right)_{u u}^{\tilde{\mathcal{S}}}=\tilde{\mathcal{F}}_{u u}^{\mathcal{S}}\left(R^{*}, E^{*}\right)_{d u^{\prime}}^{\tilde{\mathcal{S}}} \\
& \left(R^{*}, E^{*}\right)_{d d}^{\tilde{\mathfrak{S}}}=\tilde{\mathcal{F}}_{d d}^{\mathfrak{S}}\left(R^{*}, E^{*}\right)_{d d}^{\tilde{\mathfrak{S}}}=\tilde{\mathcal{F}}_{d d}^{\mathfrak{S}}\left(R^{*}, E^{*}\right)_{u d}^{\tilde{\mathfrak{S}}}, \\
& \left(R^{*}, E^{*}\right)_{u d}^{\tilde{\mathcal{S}}}=\tilde{\mathcal{F}}_{u d}^{\mathcal{S}}\left(R^{*}, E^{*}\right)_{u u}^{\tilde{\mathfrak{S}}}=\tilde{\mathcal{F}}_{u d}^{\mathcal{S}}\left(R^{*}, E^{*}\right)_{d u^{\prime}}^{\tilde{\mathfrak{S}}} \\
& \left(R^{*}, E^{*}\right)_{d u}^{\tilde{S}}=\tilde{\mathcal{F}}_{d u}^{\mathfrak{S}}\left(R^{*}, E^{*}\right)_{d d}^{\tilde{\mathfrak{S}}}=\tilde{\mathcal{F}}_{d u}^{\mathfrak{S}}\left(R^{*}, E^{*}\right)_{u d^{\prime}}^{\tilde{\mathfrak{S}}}
\end{aligned}
$$

where subscripts $i, j \in\{u, d\}$ are shorthand for $i, j \in\left\{x^{u}, x^{d}\right\}$.

Since by assumption no constant trader is present, the excess supply of the risky asset when the shock hits is neither absorbed nor mitigated, and the effect of liquidation shocks is maximised. The price of the risky security has to adjust for the continually injected new wealth by means of the paid dividend (in analogous fashion as within steady state $\tilde{\mathbb{C}}$ ), and for the jumps in the market portfolio induced by the effect of liquidation needs. A closer inspection of Eqs. (3.10) and (3.11) reveals that the following relations hold:

$$
\begin{aligned}
r^{u d}<g<r^{d u}, \\
e^{u}<e^{d} .
\end{aligned}
$$

Intuitively, in those periods in which $x=x_{-1}$ the equilibrium locally resembles steady state $\tilde{\mathbb{C}}$, and the return must equal the rate of growth of the dividend $g$ for the said reason, irrespective of the prevailing portfolio fraction. Conversely, the return is $r^{\text {ud }}<g$ whenever the liquidation shock hits, and stochastic traders pass from a high to a low investment in the risky asset. The opposite occurs when the traders recover to the high level on investment, with $r^{d u}>g$.

When the liquidation shock hits, the capital gain $r^{u d}$ may even be negative. In particular it holds

$$
r^{u d}<0 \Longleftrightarrow g<\tilde{g}=\frac{x^{u}-x^{d}}{x^{d}\left(1-x^{u}\right)}
$$

The threshold $\tilde{g}$ below which $r^{u d}$ is negative depends inversely on the magnitude of the portfolio shift in terms of wealth, i.e. on the difference $x^{u}-x^{d}$, and is influenced by the overall position of $x^{u}$ and $x^{d}$ within the unit simplex. For an extremely mild drop of around $1 \%$ of the wealth invested in the risky security, $\min (\tilde{g}) \approx 0.04$ (and note that the function $\tilde{g}\left(x^{u}, x^{d}\right)$ is highly convex). When a more economically meaningful deviation occurs, say $x^{u}-x^{d}=0.1$, then $\min (\tilde{g}) \approx 0.49$, meaning that only a substantial dividend growth rate is required for $r^{u d}$ to be positive; such a rate is clearly unsustainable in the long run and at sharp odd with actual market data. Therefore, in most conceivable cases, in response to a downward portfolio shock, the stochastic trader has to 'fictionally' pay a significant premium in order to immediately sell part of her holdings. This is typical of a fire-sale in the sense of Shleifer and Vishny (2011). Conversely, during an upward portfolio shift, the 
stochastic trader exerts an $a b$-normal pressure on the demand of the risky security, captured in turns by a higher realised return $r^{d u}>g$, eventually driving the market-clearing price upwards. As we shall see, both movements are mitigated by the presence of so-called constant traders, as long as the latter survive in the long-run.

\section{Heterogeneous traders economy}

We can now proceed with studying the effect of liquidation shocks in an economy with heterogeneous traders, the latter partitioned into the constant group $\mathbb{C}$ and the stochastic group $\mathfrak{S}$. To this aim, we need to investigate the asymptotic survival of both groups. Should we find that the stochastic group vanishes in the long-run, then the impact of liquidation shocks would simply be transient. To this purpose, we shall use the following Terminology.

Terminology. Group $i \in\{\mathbb{C}, \mathfrak{S}\}$ is said to

$\diamond$ survive if its asymptotic wealth-share is strictly positive $\mathbb{P}$-almost surely, i.e. if $\mathbb{P}\left\{\lim _{t \rightarrow \infty} \sup \varphi_{n, t}>0\right\}=1 ;$

$\diamond$ vanish if its asymptotic wealth-share is zero $\mathbb{P}$-almost surely, i.e. if $\mathbb{P}\left\{\lim _{t \rightarrow \infty} \sup \varphi_{n, t}=0\right\}=1 ;$

$\diamond$ dominate if its asymptotic wealth-share is one $\mathbb{P}$-almost surely, i.e. if $\mathbb{P}\left\{\lim _{t \rightarrow \infty} \inf \varphi_{n, t}=1\right\}=1$.

We are interested in characterising all the possible long-run outcomes. We adapt the concepts of steady state given for homogeneous traders economies in Definition 1 and 2 to the current heterogeneous traders set-up, leading to Definition 3 and 4, respectively.

Definition 3. A deterministic steady state of system (2.15) is a vector $\left(\varphi^{*}, r^{*}, e^{*}\right) \in \mathcal{D}$ such that $\left(\varphi^{*}, r^{*}, e^{*}\right)=\mathcal{F}_{x_{-1}, x}\left(\varphi^{*}, r^{*}, e^{*}\right)$ for all $\left(x_{-1}, x\right)$ in $\times_{2}\left\{x^{u}, x^{d}\right\}$.

Definition 4. Call $\mathcal{D}^{*}$ the space of all random vectors $\left(\Phi^{*}, R^{*}, E^{*}\right): X_{2}\left\{x^{u}, x^{d}\right\} \rightarrow \mathcal{D}$. A stochastic steady state of system (2.15) is a random vector $\left(\Phi^{*}, R^{*}, E^{*}\right) \in \mathcal{D}^{*}$ such that $\left(\Phi^{*}, R^{*}, E^{*}\right)_{x_{-1}, x}=\mathcal{F}_{x_{-1}, x}\left(\Phi^{*}, R^{*}, E^{*}\right)_{x_{-2}, x_{-1}}$, for all $\left(x_{-2}, x_{-1}\right)$ and $\left(x_{-1}, x\right)$ in $X_{2}\left\{x^{u}, x^{d}\right\}$.

It is possible to show that, under the stated assumptions, system (2.15) admits exactly two steady states, one for each of the the aforementioned types. ${ }^{4}$

Proposition 3. System (2.15) admits:

$\diamond$ a deterministic steady state $\mathbb{C}$ in which the constant group dominates and the stochastic group vanishes

$$
\mathbb{C}=\left(0, g, g \frac{1-\bar{x}}{\bar{x}}\right)
$$

${ }^{4}$ With an abuse of notation we denote by $i \in\{\mathbb{C}, \mathfrak{S}\}$ the steady state in which group $i$ itself dominates. 
$\diamond$ a stochastic steady state $\mathfrak{S}$ in which the stochastic group dominates and the constant group vanishes

$$
\mathfrak{S}=\left(1, g \frac{x\left(1-x_{-1}\right)}{x_{-1}(1-x)}+\frac{x-x_{-1}}{x_{-1}(1-x)}, g \frac{1-x_{-1}}{x_{-1}}\right) .
$$

Proof. See Appendix C.

Importantly for our purposes, at steady state $\mathbb{C}$ the liquidation shocks do not lead to liquidation risk, for the fraction of wealth (and therein of risky asset) in the hands of the traders who are subject to liquidation shocks is negligible. On the contrary, at steady state $\mathfrak{S}$ liquidation shocks do lead to a systemic liquidation risk, since now it is the fraction of traders who are not subject to liquidation shocks that is negligible, in aggregate wealth terms. In what follows we shall investigate the convergence to this two scenarios as well as the case in which both groups of agents survive. In the latter case, liquidation risk is endogenous in that its extent and systemic nature also depend on the traders' relative wealth dynamics.

\subsection{Sufficient conditions for no liquidation risk}

Here we investigate the conditions that prompt the constant group to dominate in the long-run, and consequently the stochastic group and associated liquidation risk to vanish.

In order to characterise the asymptotic stability of this steady state, ${ }^{5}$ we restrict to its local analysis for tractability reasons. The following Lemma is useful.

Lemma 1. The local asymptotic stability of steady state $\mathbb{C}$ is uniquely determined by the value of the (gross) growth rate $\left.\frac{\partial \varphi}{\partial \varphi_{-1}}\right|_{\mathbb{C}}$ of the wealth share $\varphi$, computed at the equilibrium.

Proof. See Appendix D.

Let us define $\left.\rho^{i}\right|_{j}$ as the expected (gross) growth rate of the wealth share of group $i$ when the system is at the steady state $j$, with $i, j \in\{\mathbb{C}, \mathfrak{S}\}$. The following Proposition provides sufficient conditions for the local asymptotic stability of steady state $\mathbb{C}$.

Proposition 4. If parameters $\bar{x}, x^{u}, x^{d}, g, \pi^{u}$, and $\pi^{d}$ are such that the following condition holds

$$
\left(1+g \frac{x^{u}}{\bar{x}}\right)^{\frac{\pi^{u}}{\pi^{u}+\pi^{d}}}\left(\bar{x}+g \frac{x^{d}}{\bar{x}}\right)^{\frac{\pi^{d}}{\pi^{u}+\pi^{d}}}=\left.\rho^{\mathfrak{S}}\right|_{\mathfrak{C}}<\left.\rho^{\mathbb{C}}\right|_{\mathbb{C}}=1+g,
$$

then steady state $\mathbb{C}$ is locally asymptotically stable, whereas if $\left.\rho^{\mathfrak{S}}\right|_{\mathbb{C}}>\left.\rho^{\mathbb{C}}\right|_{\mathbb{C}}$ then $\mathbb{C}$ is unstable.

Proof. See Appendix E.

By restricting to a local analysis, we are evaluating the conditions for the stochastic group, and hereby of the liquidation risk, to survive when returns are determined by constant

\footnotetext{
${ }^{5}$ For random dynamical systems, a steady state (either deterministic or stochastic) is asymptotically stable when, for $\mathbb{P}$-almost all sequences of random shocks $\left\{x_{t}, t \in \mathbb{N}\right\}$, the path of states generated by the composition of maps $\tilde{\mathcal{F}}_{x_{t-1}, x_{t}}^{\mathfrak{S}} \circ \cdots \circ \tilde{\mathcal{F}}_{x_{0}, x_{1}}^{\mathfrak{S}}$ converges to it, provided the initial state is chosen close enough.
} 
traders. In this case the stochastic traders hold a tiny fraction of wealth and liquidation needs are perfectly absorbed by the market. In other words, no fire-sale phenomenon occurs. The same happens for price increases that follow the recovery of the stochastic traders' positions. No such price upsurge occurs because the fraction of stochastic traders triggering them is too small. Under these conditions, if $\bar{x} \geq x^{u}$ then the constant traders enjoy a higher growth for all realizations of the shock. Inequality (4.3) is always satisfied and the steady state is locally asymptotically stable. Conversely, if $\bar{x} \leq x^{d}$ then the constant traders experience a lower growth with probability one, and the steady state is unstable. In a locally asymptotically stable equilibrium the survivor group must invest a higher share of wealth in the risky asset with respect to the vanisher. Intuitively, were it not the case, for the risky security yields a higher return with respect to the bond, an arbitrarily small redistribution in favour of the vanisher would bring the system further and further away from the initial steady state, implying the latter is unstable. The results presented here are the stochastic generalisation of those already present in the literature (Anufriev and Dindo, 2010, Proposition 5.2) regarding so-called 'fundamentalist' rules.

The interesting case is when $x^{d}<\bar{x}<x^{u}$ holds. Whenever $x_{-1}=x^{u}$ the wealth share $\varphi$ of the stochastic traders increases, i.e. $\frac{\partial \varphi}{\partial \varphi_{-1}}>1$; conversely, whenever $x_{-1}=x^{d}, \varphi$ shrinks, i.e. $\frac{\partial \varphi}{\partial \varphi_{-1}}<1$. The geometric average of growth rates ultimately determines the relevant long-run outcomes. Coteris paribus, the larger $\pi^{d}$, or the smaller $\pi^{u}$, the higher the likeliness that condition (4.3) is satisfied.

In general, it is not possible to obtain a closed form solution of the inequality in (4.3) in terms of $\bar{x}$ since $\left.\rho^{\mathfrak{S}}\right|_{\mathbb{C}}$ is not algebraic. We are only able to analytically work out the following:

Special case. $\pi^{u}=\pi^{d}$

When the transition probabilities coincide, implying that the average duration of aggressive investment equals that of modest investment, inequality (4.3) translates into the following algebraic condition in terms of the constant trader portfolio rule:

$$
\begin{aligned}
& \bar{x}>\frac{x^{u}+x^{d}+\sqrt{4 g x^{u} x^{d}(2+g)+\left(x^{u}+x^{d}\right)^{2}}}{2 g+4} \\
&=\frac{x^{u}+x^{d}}{2}-h(g), \\
& \text { with } \quad h(g) \geq 0, \quad h(0)=0, \quad h^{\prime}(g)>0 .
\end{aligned}
$$

The last equality shows that the RHS of inequality. (4.4) converges from below to the simple arithmetic average between $x^{u}$ and $x^{d}$, as $g$ approaches zero.

This result implies that there would indeed be loss of generality should one adopt the socalled deterministic skeleton approach and substitute the $\left\{x_{t}\right\}$ process with its (geometric) expected value. The noise component incorporated into the stochastic traders' portfolio brings a detrimental effect to their own survival, at least in the $\pi^{u}=\pi^{d}$ case, since the constant traders can dominate even investing a portfolio that is strictly safer than the average of the stochastic ones. In other words, the results of Anufriev et al. (2006) and Anufriev 
and Dindo (2010) regarding the asymptotic dominance of fixed-mix strategies are not alone sufficient to infer the long-run dynamics if at least one of the portfolios incorporate some noise.

The following Proposition provides a sufficient condition for the dominance of the constant trader even when $\pi^{u} \neq \pi^{d}$.

Proposition 5. If $\bar{x} \geq \mathbb{E}\left[x_{t}\right]$ then steady state $\mathbb{C}$ is locally asymptotically stable.

Proof. See Appendix F.

Importantly, for liquidation shocks to turn into liquidation risk, the stochastic traders must be, on average, more 'aggressive' than the rest of the traders.

Finally, it is possible to show that there always exists exactly one value $x^{\prime}$ of $\bar{x}$ where a bifurcation occurs. Moreover, such value is bounded from below by $x^{d}$ and from above by $x^{u}$.

Proposition 6. $\exists ! x^{\prime} \in(0,1)$ such that steady state $\mathbb{C}$ is locally asymptotically stable $\forall \bar{x}>x^{\prime}$ and is unstable $\forall \bar{x}<x^{\prime}$. Moreover, the following relation holds:

$$
x^{d}<x^{\prime}<x^{u} .
$$

Proof. See Appendix G.

\subsection{Sufficient conditions for systemic liquidation risk}

Here we investigate the conditions that prompt the stochastic group to dominate, so that liquidation shocks turn, in the long run, into the systemic liquidation risk implied by the stochastic steady state $\mathfrak{S}$. As before, for tractability reasons, we proceed with the stability analysis for initial wealth distribution skewed in favour of the stochastic group.

Corollary 1 is useful in order to derive the condition for local asymptotic stability of steady state $\mathfrak{S}$ in Proposition 7.

Proposition 7. If parameters $\bar{x}, x^{u}, x^{d}, g, \pi^{u}$, and $\pi^{d}$ are such that the following condition holds

$$
\begin{aligned}
& {\left[1+\frac{g \bar{x}}{x^{u}}\right]^{\frac{\pi^{u}\left(1-\pi^{d}\right)}{\pi^{u}+\pi^{d}}} \cdot\left[1+g \frac{\bar{x}}{x^{u}}+\frac{\left(x^{u}-\bar{x}\right)\left(x^{u}-x^{d}\right)}{x^{u}\left(1-x^{u}\right)}\right]^{\frac{\pi^{u} \pi^{d}}{\pi^{u}+\pi^{d}}}} \\
& \cdot\left[1+g \frac{\bar{x}}{x^{d}}+\frac{\left(\bar{x}-x^{d}\right)\left(x^{u}-x^{d}\right)}{x^{d}\left(1-x^{d}\right)}\right]^{\frac{\pi^{u} \pi^{d}}{\pi^{u}+\pi^{d}}} \cdot\left[1+\frac{g \bar{x}}{x^{d}}\right]^{\frac{\pi^{d}\left(1-\pi^{u}\right)}{\pi^{u}+\pi^{d}}}=\left.\rho^{\mathbb{C}}\right|_{\mathfrak{S}}<\left.\rho^{\mathfrak{S}}\right|_{\mathfrak{S}}=1+g,
\end{aligned}
$$

then steady state $\mathfrak{S}$ is locally asymptotically stable, whereas if $\left.\rho^{\mathbb{C}}\right|_{\mathfrak{S}}>\left.\rho^{\mathfrak{S}}\right|_{\mathfrak{S}}$ then $\mathbb{C}$ is unstable.

Proof. See Appendix H.

Similar to the previous case regarding steady state $\mathbb{C}$, the eigenvalue $\left.\rho^{\mathbb{C}}\right|_{\mathfrak{S}}$ is non-algebraic and therefore a closed form solution in terms of of $\bar{x}$ is in general unfeasible. The following 
Proposition derives sufficient conditions regarding stability and instability of steady state $\mathfrak{S}$ in terms of the constant group portfolio rule.

Proposition 8. Given $\pi^{u}, \pi^{d}, x^{u}$ and $x^{d}$, a sufficient condition for local asymptotic stability of steady state $\mathfrak{S}$ is

$$
\bar{x} \leq x^{d} \quad \wedge \quad g \geq \frac{x^{u}-x^{d}}{1-x^{u}}
$$

with at least one strict inequality sign. Conversely, a sufficient condition for instability of steady state $\mathfrak{S}$ is

$$
\bar{x} \geq x^{u} \quad \wedge \quad g \leq \frac{x^{u}-x^{d}}{1-x^{u}}
$$

with at least one strict inequality sign.

Proof. See Appendix I.

Proposition 8 also sheds some light on the role played by the dividend rate of growth. From Eq. (2.11) and the first equation of system (2.15) it is clear that, coeteris paribus, the higher $g$, the higher the dividend yield and, in turns, the greater the wealth share of the group which is more exposed towards the risky security.

The following Conjecture, based on a numerical investigation of the parameters space, is the analogous of Proposition 6 regarding steady state $\mathfrak{S}$.

Conjecture 1. $\exists ! x^{\prime \prime} \in(0,1)$ such that steady state $\mathfrak{S}$ is locally asymptotically stable $\forall \bar{x}<x^{\prime \prime}$ and is unstable $\forall \bar{x}>x^{\prime \prime}$.

Hint. See Appendix J.

\subsection{Sufficient conditions for endogenous liquidation risk}

So far we have investigated the conditions under which either no liquidation risk (Section 4.1) or systemic liquidation risk occurs asymptotically (Section 4.2). In this section we prove the existence of a non-degenerate region of the parameters space for which liquidation risk is endogenous. Given that both groups of traders survive, their relative wealth dynamics is a mean reverting process, and the transformation of liquidation shocks into liquidation risk depends on the fraction of wealth in the hands of the traders subject to liquidation needs. The following Proposition provides a sufficient condition for the emergence of endogenous liquidation risk.

Proposition 9. $\exists \hat{g}>0$ such that $\forall g<\hat{g}$ the following relation holds:

$$
x^{\prime \prime}<x^{\prime}
$$

Proof. See Appendix K. 


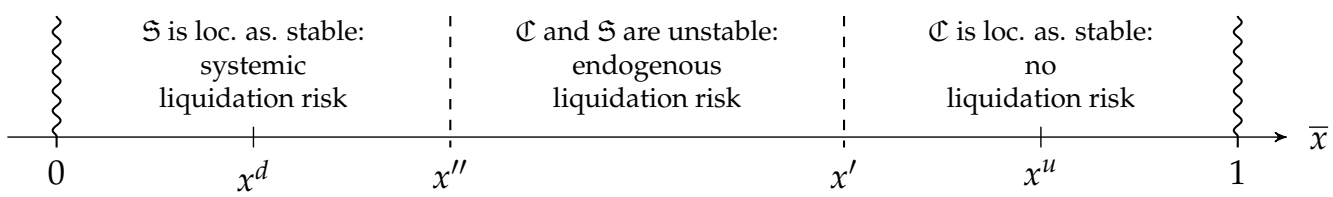

(a)

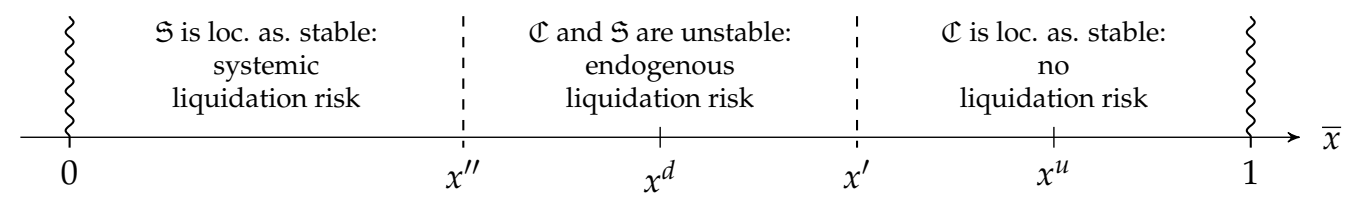

(b)

Figure 3: Stability regions of steady states $\mathbb{C}$ and $\mathfrak{S}$ and emergence of liquidation risk as a function of $\bar{x}$. (a): Regime with $x^{d}<x^{\prime \prime}$. (b): Regime with $x^{d}>x^{\prime \prime}$.

While Proposition 9 only provides a sufficient condition for the existence of long-run heterogeneity, and consequently of endogenous liquidation risk, a closer look at Eq. (4.5) reveals that the interaction between different parameters is non-trivial. For example, a certain degree of substitutability between the rate of growth of the dividend and the probability of a liquidity crisis is apparent in Eq. (K.1): depending on the relative magnitude of $\left.\rho_{u u}^{\mathbb{C}}\right|_{\mathfrak{S}}\left(x^{d}\right)$ and $\left.\rho_{u d}^{\mathbb{C}}\right|_{\mathfrak{S}}\left(x^{d}\right)$ there might exists $\hat{\pi}^{d} \in(0,1)$ such that $\forall \pi^{d}>\hat{\pi}^{d}$ the relation $x^{\prime \prime}<x^{\prime}$ holds even though $g \geq \hat{g}$. A numerical inspection of the parameters space validates the following Conjecture.

Conjecture 2. The following relation holds $\forall g>0$ :

$$
x^{\prime \prime}<x^{\prime}
$$

Hint. See Appendix L.

Fig. 3 portrays the relevant findings about the emergence of liquidation risk for all the steady states.

The position of the threshold value $x^{\prime \prime}$ may be either to the left or to the right of $x^{d}$, depending on the underlying parametrisation of the model. Following Proposition 9, if $g<\hat{g}$ then $x^{\prime \prime}<x^{d}$. Remarkably, when this is the case, liquidity risk is endogenous. The constant group is able to invade, albeit not to dominate, the stochastic one even though the demand of the constant traders is always lower than that of the stochastic traders, i.e. when the relation $x^{\prime \prime}<\bar{x}<x^{d}$ holds. Along these equilibrium paths, losses due to fire-sales are particularly severe.

\section{Simulations and sensitivity analysis}

In the previous Section we have outlined a liquidation risk taxonomy of all the long-run outcomes that system (2.15) admits. In this section, we focus on the case where liquidation 


\begin{tabular}{lcc}
\hline Description & Variable & Value \\
\hline dividend rate of growth & $g$ & 0.05 \\
constant investment & $\frac{\delta}{x}$ & 0.45 \\
stochastic investment up & $x^{u}$ & 0.7 \\
stochastic investment down & $x^{d}$ & 0.3 \\
probability down when up & $\pi^{d}$ & 0.01 \\
probability up when down & $\pi^{u}$ & 0.01 \\
initial wealth share & $\varphi_{0}$ & 0.5 \\
initial return & $r_{0}$ & null \\
initial yield & $e_{0}$ & 0.01 \\
\hline
\end{tabular}

Table 1: Parameters value and initial conditions.

risk is endogenous and we investigate its main determinants in terms of the interaction between the underlying parameters of the economy.

\subsection{Simulations}

Following Fig. 3, we distinguish two main regimes. In the first, the relation $x^{\prime \prime}>x^{d}$ holds, and the constant traders need to invest $\bar{x} \in\left(x^{d}, x^{u}\right)$ in order to invade, let alone dominate, the stochastic group and thus mitigate the transformation of liquidation shocks into liquidation risk. In the second regime instead $x^{\prime \prime}<x^{d}$, and the constant traders are able to invade, although not to dominate, by investing a strictly less fraction of their wealth in the risky asset. Albeit the long-run outcome of the two regimes is analytically analogous, the economic interpretation of the inherent trade-off is quite different. Fig. 4 portrays a typical simulation run within the first regime (cf. Fig. 3). The model is parametrised according to Table 1 . To ensure a situation of long-run heterogeneity, the value of $\bar{x}$ is selected close to the mid-point between the thresholds $x^{\prime}$ and $x^{\prime \prime}$, the latter being evaluated numerically. The first panel depicts the portfolio fraction $\bar{x}$ of the constant group (green) and a realisation of the stochastic group $\left\{x_{t}\right\}$ process (blue). The second panel reports the dynamics of the wealth share $\varphi_{t}$. At the beginning of the run, the overall endowment is evenly split between the two groups. As trading unfolds, wide and sharp fluctuations in the distribution of aggregate wealth appear and persist indefinitely, as the system never converges towards a steady state. We find that the value of the growth rate of the dividend is largely responsible for this wild dynamics. In general, the higher $g$, the wider the fluctuations of $\varphi_{t}$. In both the price (third panel) ${ }^{6}$ and return (fourth panel) series, a clear pattern emerges when compared to the wealth share dynamics. When $\varphi_{t}$ is large, both prices and returns are highly volatile; conversely, when $\varphi_{t}$ is small, $p_{t}$ and $r_{t}$ are relatively stable. Intuitively, when the stochastic group controls most of the wealth in the economy, it has a great impact on the market portfolio, and eventually on the clearing price. When this is the case, the regime shifts implied by the Markov process $\left\{x_{t}\right\}$ are to a large extent incorporated into the prevailing price and the liquidation risk is high. This pattern closely resembles the famous volatility clustering stylised fact, i.e. the observation

\footnotetext{
${ }^{6}$ To get a more appreciable picture, the price reported in the third panel has been discounted by its nonstationary component $1+g$.
} 

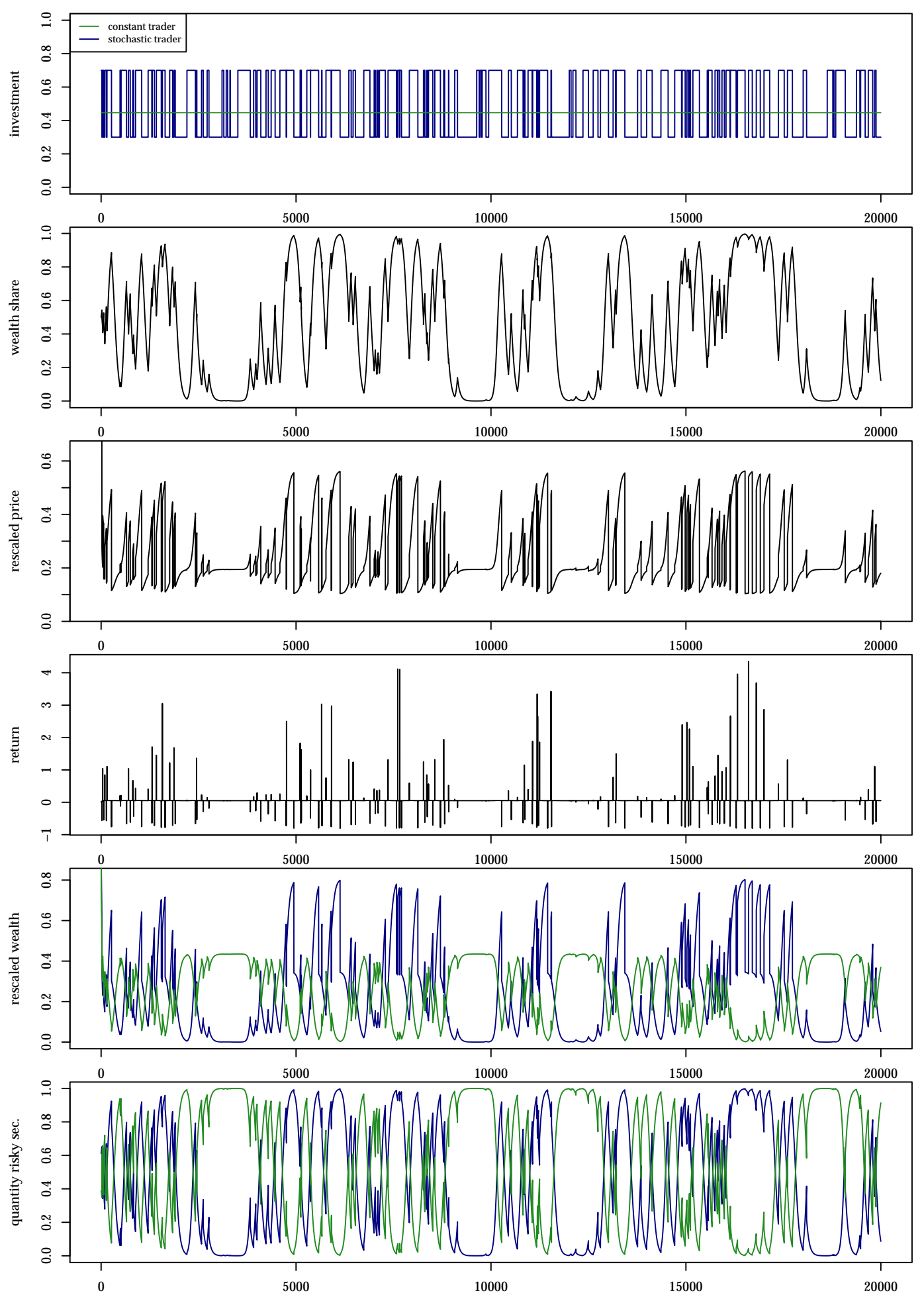

Figure 4: Typical simulation run within the first regime (cf. Fig. 3). Green and blue series refer to the constant and stochastic stochastic trader variables, respectively. Initialisation as of Table 1. 
that 'large changes tend to be followed by large changes, of either sign, and small changes tend to be followed by small changes' (see Mandelbrot, 1963). A similar argument goes for the dynamics of traders' level of wealth (fifth panel) ${ }^{7}$ and actual exchanged quantity of the risky asset (sixth panel). When the stochastic group is predominantly influential, the system locally resembles steady state $\mathfrak{S}$ where prices, and hence the market value of the portfolio, fluctuate according to the aforementioned stochastic 4-cycle (cf. Fig. 2(b) and Corollary 1) while a null quantity of the asset is actually exchanged.

As representative of the second regime, Fig. 5 shows the same simulation as before (i.e. with the same seed), but featuring a lower rate of growth of the dividend and the constant portfolio is always strictly less than the stochastic one. In particular, it is $g=0.005$ and $\bar{x}=0.25$ (close to the midpoint between $x^{\prime \prime}$ and $x^{d}$ ), while everything else remains as of Table 1. The fluctuations in the wealth share (second panel) appear dampened compared to Fig. 4. Intuitively, $g$ has a positive influence on the speed of wealth adjustment for the group holding the greatest part of wealth invested in the risky security. Moreover, the wealth share itself remains, on average, relatively high; after all, the stochastic trader is exploiting a strong arbitrage at every time step (cf. Assumption 1), and more than the constant trader does. Consequently, the volatility of all the ensuing series is necessarily more persistent. In the fifth panel it is also clearly visible that the randomness incorporated in the stochastic traders behaviour generates an externality since it not only exerts an effect on the market value of their own portfolio through its influence on the prevailing price, but also on the level of wealth of the constant group. That said, what really makes a difference from an economic point of view with respect to the previous scenario lies in the response of the system in terms of wealth allocation to the local realisation of the Markov process $\left\{x_{t}\right\}$. As already shown, the dynamics of the economy locally depends on two consecutive realisations of the stochastic process (cf. system 2.15). Fig. 6 provides a close-up of both the simulations presented before, covering a shorter period for visibility purposes. In both the scenarios, as long as $x_{t-1}=x_{t}=x^{u}$ the wealth of the stochastic group grows faster than that of the constant group, since $x^{u}>\bar{x}$; as a result, the wealth share goes up. When the stochastic portfolio suddenly shifts from high to low investment in the risky security, i.e. when $x_{t-1}=x^{u}$ and $x_{t}=x^{d}$, a fire-sale phenomenon puts downward pressure on the price and consequently reduces the level of wealth of both the groups, proportionally on their relative positions at time $t-1$. Again, since $x^{u}$ is always greater than $\bar{x}$, the deflation hits the stochastic group to a larger extent, and in both cases the wealth share plummets accordingly. During the phases in which $x_{t-1}=x_{t}=x^{d}$, instead, the stochastic group is worse off in the first scenario, since $x^{d}<\bar{x}$, and better off in the second, where the opposite relation holds true. Hence, the wealth share shrinks in the left panel and expands in the right one. For the same reason, when the stochastic portfolio suddenly shifts from low to high investment in the risky security, i.e. when $x_{t-1}=x^{d}$ and $x_{t}=x^{u}$, the ensuing inflation favours the stochastic group in the second scenario but penalises it in the first. Therefore, the wealth shares jump downwards in the first regime and upwards in the second. ${ }^{8}$

\footnotetext{
${ }^{7}$ Again, for representational purposes, the series have been made stationary by discounting by $1+g$.

${ }^{8}$ Within the second regime, $\bar{x}=x^{d}$ constitutes a special case in which no jump occurs when $\left(x_{t-1}, x_{t}\right)=\left(x^{d}, x^{u}\right)$ and the wealth share is constant whenever $x_{t-1}=x_{t}=x^{d}$.
} 

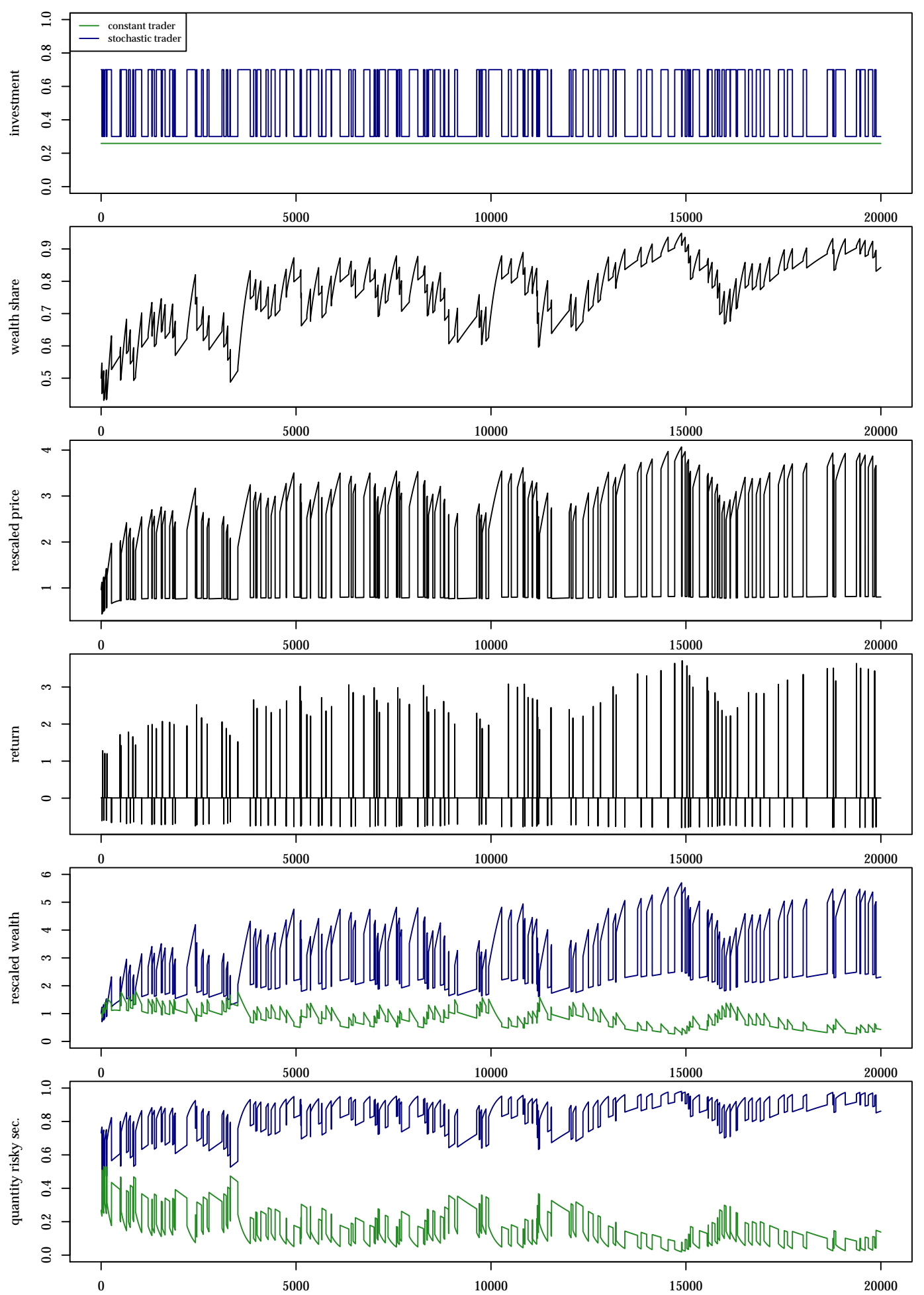

Figure 5: Typical simulation run within the second regime (cf. Fig. 3). Green and blue series refer to the constant and stochastic stochastic trader variables, respectively. Initialisation as of Table 1 except $g=0.005$ and $\bar{x}=0.25$. 

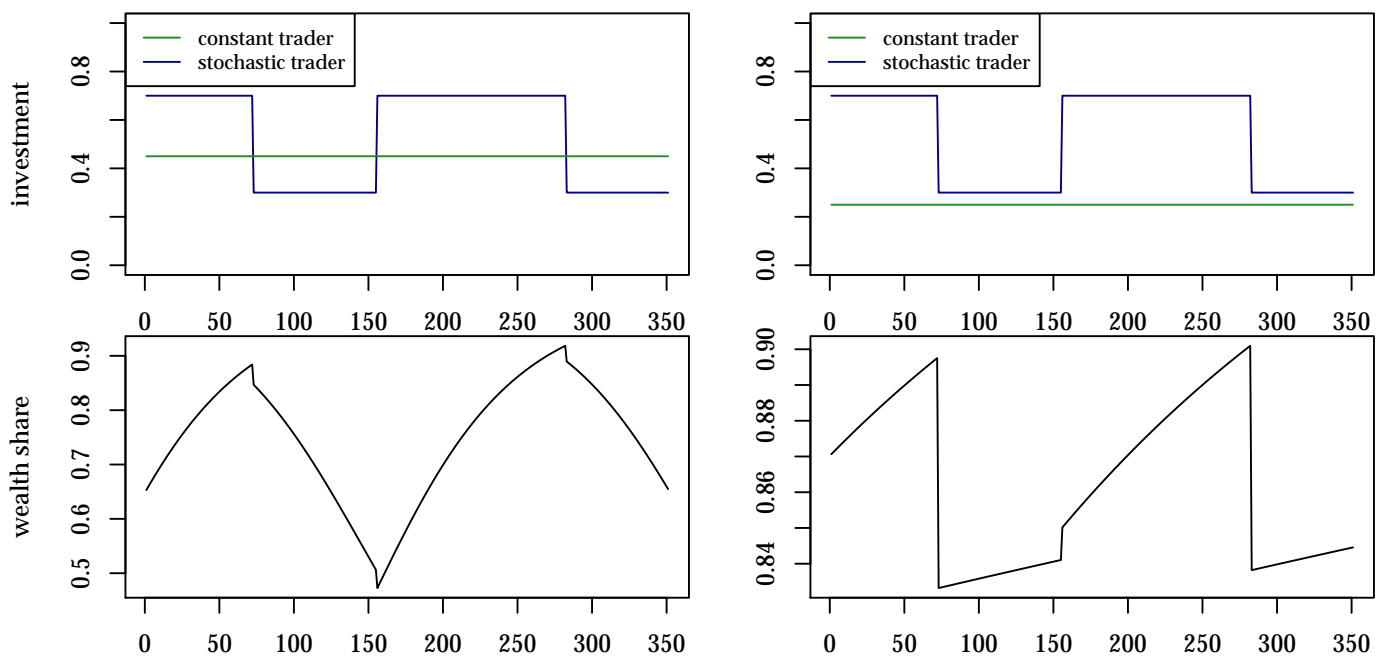

Figure 6: Close-up of simulations in Fig. 4 (left) and Fig. 5 (right).

Unsurprisingly, within the second regime, whenever $x_{t-1}=x_{t}$ the stochastic traders outperform the constant ones, irrespectively of whether they invest $x^{u}$ or $x^{d}$. But then, how comes that the stochastic group does not dominate in the long run? Notice that both the speed of adjustment during recurrence phases (when $x_{t-1}=x_{t}$ ) and the magnitude of the jumps during transience phases (when $x_{t-1} \neq x_{t}$ ) are positively related, cœteris paribus, to the relative distance between the traded portfolios. Intuitively, the larger $\left|x_{t}-\bar{x}\right|$, the higher the dividend gain during recurrence phases for the group holding the greatest position in the risky asset, and the higher the excess demand/supply during transience phases, in turns translating into a greater absolute return. Since the difference $x^{u}-\bar{x}$ is necessarily larger in the second scenario, the continuous wealth rebalancing takes place by means of smoothly increasing and decreasing recurrence phases in the first regime, whilst it occurs as sharp, discrete downward jumps in the market clearing price in the second. Apparently, the very nature of the underlying trade-off differs in the two cases.

\subsection{Sensitivity analysis}

We are left with studying to what extent changes in the values of the underlying parameters influence the asymptotic dynamics of the economy. Starting from the usual parametrisation of Table 1, Fig. 7 shows the evolution of the threshold values $x^{\prime}$ and $x^{\prime \prime}$ in terms of the constant trader portfolio $\bar{x}$ for changes in $g$ (top-left panel), in the position of $x_{t}$ within the unit simplex with constant jumps $x^{u}-x^{d}$ (top-right), in the dispersion of $x_{t}$ around a constant mean (bottom-left), and in the transition probability $\pi$, under the special case $\pi=\pi^{u}=\pi^{d}$ (bottom-right). In each plot, the relative position of the two thresholds splits the Carthesian plane in three distinct areas, corresponding to the stable regions of both steady state $\mathbb{C}$ and $\mathfrak{S}$, and their jointly unstable region where long-run heterogeneity, and herein endogenous liquidation risk, occur.

From the top-left panel, we can see that $g$ has a (slightly) negative effect on the threshold 

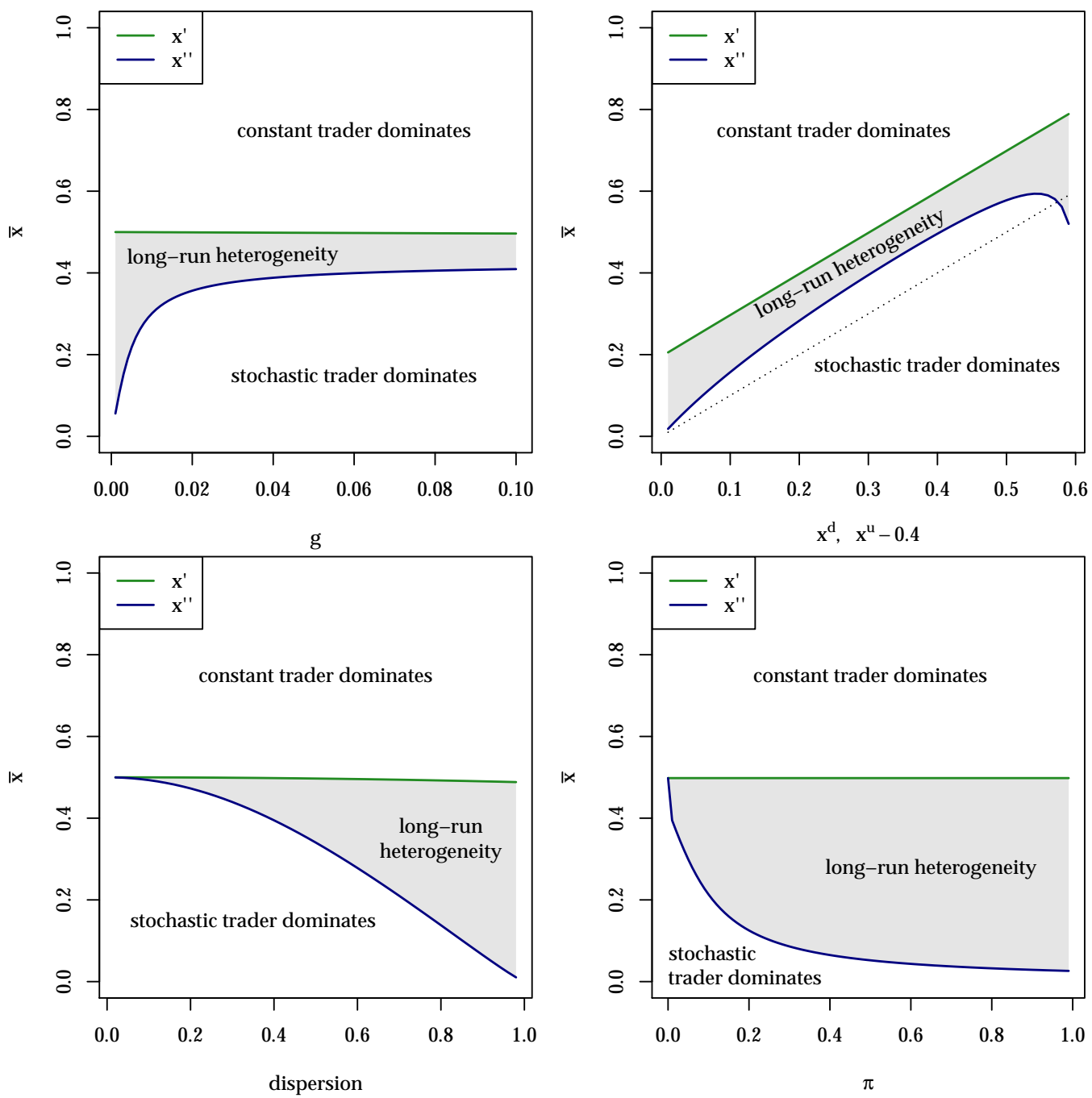

Figure 7: Sensitivity analysis. Initialisation as of Table 1 when not otherwise specified. 
$x^{\prime}$ and a positive effect on $x^{\prime \prime}$; the first result has been already shown in Special case 2 and generalised in Appendix F. Therefore, the higher $g$, the narrower the interval of $\bar{x}$ for which there is long-run heterogeneity. For even higher $g$, this interval shrinks even further, and at the limit it collapses into one point:

$$
\lim _{g \rightarrow+\infty} x^{\prime}(\cdot)=\lim _{g \rightarrow+\infty} x^{\prime \prime}(\cdot)=\mathbb{E}_{\mathcal{G}}^{\pi}\left[x_{t}\right],
$$

where $\mathbb{E}_{\mathcal{G}}^{\boldsymbol{\pi}}[\cdot]$ denotes the geometric expected value with respect to the invariant distribution $\pi$ of the Markov process $\left\{x_{t}\right\}$. The top right panel shows what happens when the support of the stochastic portfolio $x_{t}$ shifts within the unit simplex, keeping the range, i.e. the extent of the liquidation need $x^{u}-x^{d}$, constant. Clearly, the width of the long-run heterogeneity corridor has a non-linear relation with $\mathbb{E}\left[x_{t}\right]$. In particular, the difference $x^{\prime}-x^{\prime \prime}$ is especially large at the extrema of the simplex. Note also that $x^{\prime \prime}(\cdot)$ is not monotone, and for sufficiently high values of $\mathbb{E}\left[x_{t}\right]$ it is decreasing. When $x^{\prime \prime}$ crosses the bisector (the dotted line, along which $\bar{x}=x^{d}$ ) from above, a non-degenerate region between the two curves appear. For any point in this region, the constant group is able to invade the stochastic group by investing a strictly less fraction of wealth in the risky asset; this is what we previously dubbed the 'second regime'. When we simulated our second scenario we opted for keeping $x^{u}$ and $x^{d}$ unchanged and reducing the dividend growth rate $g$ that, following what we said about the top-left panel, lowers the $x^{\prime \prime}$ threshold and enlarges the shaded area below the bisector line. As the top-right panel shows, however we could have kept both $g$ and the jump $x^{u}-x^{d}$ unchanged, increased $x^{d}$ close to 0.6 , and eventually end up in a mathematically equivalent dynamics. In the bottom-left panel the thresholds are plotted against the symmetric range of $x_{t}$ around a constant $\mathbb{E}\left[x_{t}\right]=0.5$. Both functions are decreasing but $x^{\prime \prime}$ is steeper, so that the interval of long-run heterogeneity widens as the dispersion increases. Intuitively, the more abrupt the change in the positions of the stochastic traders during transience phases, the more the effect of 'buying high and selling low' penalises them and favours the constant group, that is able to dominate with a lower risky position $\bar{x}$. For similar reasons, the more frequent the transience phases of $x_{t}$, the more the stochastic traders are disadvantaged. This is highlighted in the bottom-right panel, where the range of $x_{u}$ and $x^{d}$ are fixed, but $\pi=\pi^{u}=\pi^{d}$ varies. From eq. (E.1), the invariant distribution $\pi$ relative to steady state $\mathbb{C}$ always equals $\left[\frac{1}{2}, \frac{1}{2}\right]$ when $\pi^{u}=\pi^{d}$, and therefore $x^{\prime}$ is a horizontal line. The invariant distribution $\tilde{\pi}$ of $\mathfrak{S}$ in eq. (3.9) instead depends on the specific value of $\pi$. The higher $\pi$, the more likely the transience phases compared to recurrence phases, and the larger the buy-high and sell-low effect.

\section{Concluding remarks}

We investigate asset prices and wealth-driven selection in a simple financial market where traders have heterogeneous constantly rebalanced portfolios of a risk-free and a risky longlived asset, and those with the most aggressive position in the risky security are subject to joint liquidation needs. When a liquidation shock hits the economy, depending on the re- 
lative wealth of the two groups of traders, the extra supply of risky asset is either fully absorbed by the market, or it generates a fire-sale phenomenon. We provide conditions on the economy parameters such that liquidation needs are always fully absorbed in the longrun, when the stabilizing constant agents dominate, as well as conditions under which liquidation shocks always lead to a fire-sale phenomenon, when the constant agents vanish. Moreover, there exist cases when both groups survive and liquidation needs may or may not lead to a sharp drop in the risky asset price. In this case we say that liquidation risk is endogenous, its occurrence being coupled with the traders' wealth dynamics. In order to derive these results we study the random dynamical system characterizing the dynamics of relative wealth, risky asset returns, and dividend yield, as driven by an exogenous liquidation stochastic process. Our results are unobtainable from the study of the underlying deterministic skeleton alone. In particular, we show that simply taking into account the expected value of the stochastic portfolio is not sufficient to determine the long-run dynamics of the system.

Our framework can be further extended in a number of directions. A straightforward improvement is to allow for an arbitrarily large number of risky securities to be traded in the market, e.g. along the lines of the deterministic wealth selection model in Anufriev et al. (2012). This would enable the investigation of the spillover effect of liquidation shocks hitting one asset onto other assets. Another interesting dimension would be to explicitly account for the noise generated by the dividend rate of growth and to endogenise the liquidation needs based on agents expectations regarding the dividend growth rate. Finally, the investigation of more complex portfolio responses to liquidation needs, e.g. staircase adjustment, constitutes another intriguing augmentation of our framework.

\section{References}

Almazan, Andres, Keith C. Brown, Murray Carlson and David A. Chapman (2004). 'Why constrain your mutual fund manager?' Journal of Financial Economics 73(2), pp. 289-321. DOI: $10.1016 / j$.jfineco.2003.05.007.

Anufriev, Mikhail and Giulio Bottazzi (2010). 'Market Equilibria under Procedural Rationality'. Journal of Mathematical Economics 46(6), pp. 1140-1172. DOI: 10.1016/j . jmateco. 2010.09 .005$.

Anufriev, Mikhail, Giulio Bottazzi, Matteo Marsili and Paolo Pin (2012). 'Excess covariance and dynamic instability in a multi-asset model'. Journal of Economic Dynamics and Control 36(8), pp. 1142-1161. DOI: 10.1016/j . jedc.2012.03.015.

Anufriev, Mikhail, Giulio Bottazzi and Francesca Pancotto (2006). 'Equilibria, stability and asymptotic dominance in a speculative market with heterogeneous traders'. Journal of Economic Dynamics and Control 30, pp. 1787-1835. DOI: 10.1016/j . jedc. 2005.10.012.

Anufriev, Mikhail and Pietro Dindo (2010). 'Wealth-driven selection in a financial market with heterogeneous agents'. Journal of Economic Behavior \& Organization 73, pp. 327-358. DOI: $10.1016 / j$. jebo.2009.11.006. 
Black, Fischer (1986). 'Noise'. Journal of Finance 41(3), pp. 529-543. DOI: 10.1111/ j .15406261.1986. tb04513.x.

Bottazzi, Giulio and Pietro Dindo (2014). 'Evolution and market behavior with endogenous investment rules'. Journal of Economic Dynamics and Control 48, pp. 121-146. DOI: 10. 1016/j.jedc.2014.08.012.

Brock, William A. and Cars H. Hommes (1998). 'Heterogeneous beliefs and routes to chaos in a simple asset pricing model'. Journal of Economic Dynamics and Control 22(89), pp. 1235-1274. DOI: 10.1016/S0165-1889(98)00011-6.

Caballero, Ricardo J. and Alp Simsek (2013). 'Fire Sales in a Model of Complexity'. The Journal of Finance 68(6), pp. 2549-2587. DOI: 10.1111/jofi.12087.

Chiarella, Carl (1992). 'The dynamics of speculative behaviour'. Annals of Operations Research 37(1), pp. 101-123. DOI: 10.1007/BF02071051.

Chiarella, Carl, Roberto Dieci and Laura Gardini (2006). 'Asset price and wealth dynamics in a financial market with heterogeneous agents'. Journal of Economic Dynamics and Control 30(9-10), pp. 1755-1786. DOI: 10.1016/j. jedc. 2005.10.011.

Chiarella, Carl and Xue-Zhong He (2001). 'Asset Price and Wealth Dynamics Under Heterogeneous Expectations'. Quantitative Finance 1(5), pp. 509-526. DOI: 10. 1088/1469$7688 / 1 / 5 / 303$

Chiarella, Carl, Xue-Zhong He and Min Zheng (2011). 'An analysis of the effect of noise in a heterogeneous agent financial market model'. Journal of Economic Dynamics and Control 35(1), pp. 148-162. DOI: 10.1016/j. jedc.2010.09.006.

Coayla-Teran, Edson A. and Paulo R. Ruffino (2004). 'Stochastic versions of HartmanGrobman theorems'. Stochastics and Dynamics 4(4), pp. 571-593. DOI: 10 . $1142 /$ S0219493704001206.

Coval, Joshua and Erik Stafford (2007). 'Asset fire sales (and purchases) in equity markets'. Journal of Financial Economics 86, pp. 479-512. DOI: 10.1016/j . j fineco. 2006.09.007.

Evstigneev, Igor, Thorsten Hens and Klaus Reiner Schenk-Hoppé (2011). 'Local stability analysis of a stochastic evolutionary financial market model with a risk-free asset'. Mathematics and Financial Economics 5(3), pp. 185-202. DOI: 10.1007/s11579-011-0056-z.

Hommes, Cars H. (2006). 'Heterogeneous Agent Models in Economics and Finance'. In: Handbook of Computational Economics, volume 2. Ed. by Leigh Tesfatsion and Kenneth L. Judd. North-Holland, pp. 1109-1186. DOI: 10.1016/S1574-0021 (05)02023-X.

Mandelbrot, Benoit B. (1963). 'The Variation of Certain Speculative Prices'. Journal of Business 36(4), pp. 394-419. DOI: 10.1086/294632.

Mulvey, John M. and Woo Chang Kim (2010). 'Fixed Mix Strategy'. In: Encyclopedia of Quantitative Finance. Ed. by Rama Cont. Wiley. DOI: 10.1002/9780470061602. eqf14010. 
Pulvino, Todd C. (1998). ‘Do Asset Fire Sales Exist? An Empirical Investigation of Commercial Aircraft Transactions'. The Journal of Finance 53(3), pp. 939-978. DOI: 10.1111/00221082.00040.

Shleifer, Andrei and Robert Vishny (2011). 'Fire Sales in Finance and Macroeconomics'. Journal of Economic Perspectives 25(1), pp. 29-48. DOI: 10.1257/jep. 25.1.29.

Wagner, Wolf (2011). 'Systemic Liquidation Risk and the Diversity-Diversification TradeOff'. Journal of Finance 66(4), pp. 1141-1175. DOI: 10.1111/j.1540-6261.2011.01666.x.

\section{Appendices}

\section{A. Proof of Proposition 1}

Recall, first of all, that a null dividend yield is ruled out by our phase space, and therefore the trivial steady state $(0,0)$ is not applicable. The map (3.2) admits a unique non-trivial steady state

$$
e^{*}=g \frac{1-\bar{x}}{\bar{x}} .
$$

Substituting $e^{*}$ into the first equation of system (3.1) yields the equilibrium return $r^{*}=g$. It is also possible to show that:

$\diamond$ the map $f^{\tilde{\mathbb{C}}}(\cdot)$ is strictly increasing over $\mathbb{R}_{++}$since

$$
\left(f^{\tilde{\mathbb{C}}}\right)^{\prime}(\cdot)=\frac{(\bar{x}-1)^{2}}{\left[1+\bar{x}\left(e_{t-1}-1\right)\right]^{2}}(1+g)>0 ;
$$

$\diamond$ the slope of $f^{\tilde{\mathbb{C}}}$ at the steady state lies within the unit circle since

$$
\left(f^{\tilde{\mathbb{C}}}\right)^{\prime}\left(e^{*}\right)=\frac{1}{1+g} \in(0,1) .
$$

Therefore, steady state $\tilde{\mathbb{C}}=\left(g, g \frac{1-\bar{x}}{\bar{x}}\right)$ is the unique, globally stable steady state of the system.

\section{B. Proof of Proposition 2}

Recall, first of all, that a null dividend yield is ruled out by our phase space, and therefore the trivial (deterministic) steady state $(0,0)$ is not applicable. We shall show that the 1 dimensional map (3.5) admits a unique globally stable stochastic steady state. In fact, 
defining

$$
e^{u}=g \frac{1-x^{u}}{x^{u}} \quad \text { and } \quad e^{d}=g \frac{1-x^{d}}{x^{d}},
$$

as in (3.11), it holds

$$
\begin{aligned}
& e^{u}=f_{d u}^{\tilde{\mathfrak{S}}}\left(e^{d}\right)=f_{\mathcal{u} u}^{\tilde{\mathfrak{S}}}\left(e^{u}\right), \\
& e^{d}=f_{u d}^{\tilde{\mathfrak{S}}}\left(e^{u}\right)=f_{d d}^{\tilde{\mathfrak{S}}}\left(e^{d}\right) .
\end{aligned}
$$

Therefore, the state $e^{\tilde{5}}\left(x_{-1}, x\right)=g \frac{1-x_{-1}}{x_{-1}}$ is a stochastic steady state of the map (3.5). To show uniqueness and stability we shall use the following properties:

$\diamond$ for every couple $\left(x_{t-2}, x_{t-1}\right) \in X_{2}\left\{x^{u}, x^{d}\right\}$ the map $f_{x_{t-2}, x_{t-1}}^{\tilde{\mathfrak{s}}}$ is both strictly increasing,

$$
\left(f_{x_{t-2}, x_{t-1}}^{\tilde{\mathrm{S}}}\right)^{\prime}(\cdot)=\frac{x_{t-2}\left(x_{t-2}-1\right)\left(x_{t-1}-1\right)}{x_{t-1}\left[1+x_{t-2}\left(e_{t-1}-1\right)\right]^{2}}(1+g)>0,
$$

and concave over $\mathbb{R}_{++}$,

$$
\left(f_{x_{t-2}, x_{t-1}}^{\tilde{\mathfrak{y}}}\right)^{\prime \prime}(\cdot)=-\frac{2\left(x_{t-2}\right)^{2}\left(1-x_{t-2}\right)\left(1-x_{t-1}\right)}{x_{t-1}\left[1+x_{t-2}\left(e_{t-1}-1\right)\right]^{3}}(1+g)<0 .
$$

$\diamond$ for every couple $\left(x_{t-2}, x_{t-1}\right) \in X_{2}\left\{x^{u}, x^{d}\right\}$

$$
\left.\left(f_{x_{t-2}, x_{t-1}}^{\tilde{\mathcal{S}}}\right)^{\prime}(\cdot)\right|_{e=e^{x_{t-2}}}=\frac{1}{1+g} \in(0,1),
$$

where $e^{x_{t-2}}=e^{u}$ when $x_{t-2}=x^{u}$ and $e^{x_{t-2}}=e^{d}$ when $x_{t-2}=x^{d}$.

Uniqueness Given monotonicity and concavity, there exists only one $\bar{e}$ such that $f_{u u}(\bar{e})=$ $\bar{e}$. It follows that the stochastic steady state is unique.

Global stability For every $t$ and for every realisation of the Markov process $\left\{x_{t}\right\}$ the composition of maps

$$
f_{x_{t-2}, x_{t-1}}^{\tilde{\mathcal{S}}} \circ \cdots \circ f_{x_{0}, x_{1}}^{\tilde{\mathcal{S}}}
$$

is monotone and concave because it is the composition of monotone and concave maps. Moreover, due to (B.1), (B.2), and (B.5)

$$
\left.\left(f_{x_{t-2}, x_{t-1}}^{\tilde{\mathfrak{S}}} \circ \ldots \circ f_{x_{0}, x_{1}}^{\tilde{\mathfrak{S}}}\right)^{\prime}\right|_{e=e^{x_{0}}}=\left(\frac{1}{1+g}\right)^{t} .
$$


It follows that for all initial dividend yields in the open interval $\left(0, e^{d}\right)$ the dynamics converges to $e^{\tilde{5}}$ from below, whereas for all initial dividend yields in the open interval $\left(e^{d}, \infty\right)$, the dynamics converges to $e^{\tilde{5}}$ from above. Note that the convergence does not depend on $\mathbb{P}$.

Having proved the global stability of $e^{\tilde{s}}$ for the map $f$ in (3.5), the globaly stability of $\tilde{\mathfrak{s}}$ for the map $\mathcal{F}$ in (3.4) follows from the implications of the dynamics of the dividend yield for the dynamics of returns, first equation in (3.4).

\section{Proof of Proposition 3}

Steady states $\mathbb{C}$ and $\mathfrak{S}$ are straightforward generalisations of steady states $\tilde{\mathbb{C}}$ and $\tilde{\mathfrak{S}}$ derived in Proposition 1 and Proposition 2 when border conditions $\varphi^{*}=0$ and $\varphi^{*}=1$ are, respectively, imposed. It suffices to show that there exists no steady state other than the aforementioned. Hence, consider the case $\varphi^{*} \in(0,1)$ : the first equation of system (2.15) reduces to

$$
x_{-1}=\varphi^{*} x_{-1}+\left(1-\varphi^{*}\right) \bar{x} .
$$

Under Assumption 2 this condition is never satisfied since $x^{d} \neq x^{u}$. Therefore there exist no steady states other than those found above.

\section{Proof of Lemma 1}

Consider the Jacobian matrix $\mathcal{J}^{\mathbb{C}}$ of system (2.15) computed at the steady state $\mathbb{C}$

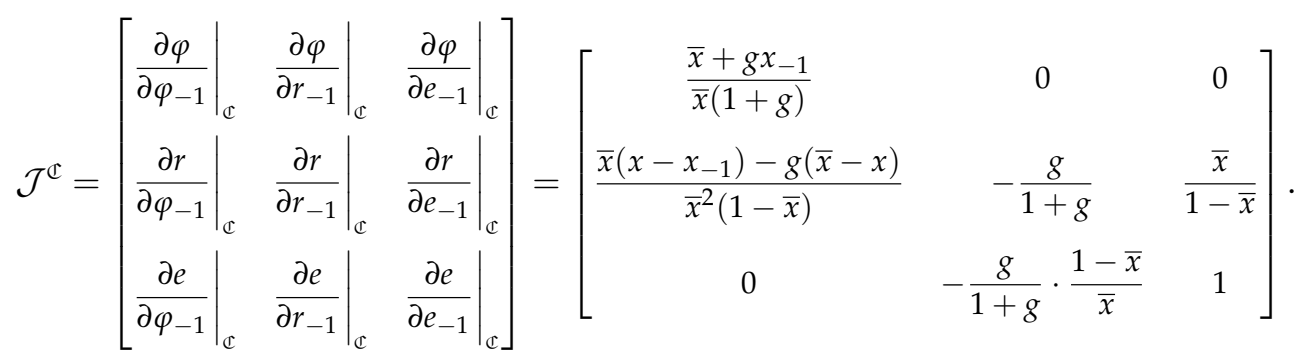

The matrix is stochastic since it depends on two consecutive realisations $x_{-1}$ and $x$ of the liquidity trader's portfolio position. Moreover, it displays the following structure:

$$
\mathcal{J}^{\mathbb{C}}=\left[\begin{array}{ll}
\boldsymbol{Q} & 0 \\
\bullet & \boldsymbol{0}
\end{array}\right],
$$


where

$$
\boldsymbol{q}=\left[\left.\frac{\partial \varphi}{\partial \varphi_{-1}}\right|_{\mathbb{C}}\right], \quad \mathbf{0}=\left[\begin{array}{ll}
0 & 0
\end{array}\right], \quad \boldsymbol{\nabla}=\left[\begin{array}{c}
\left.\frac{\partial r}{\partial \varphi_{-1}}\right|_{\mathbb{C}} \\
0
\end{array}\right], \quad \boldsymbol{\uparrow}=\left[\begin{array}{cc}
\left.\frac{\partial r}{\partial r_{-1}}\right|_{\mathbb{C}} & \left.\frac{\partial r}{\partial e_{-1}}\right|_{\mathbb{C}} \\
\left.\frac{\partial e}{\partial r_{-1}}\right|_{\mathbb{C}} & \left.\frac{\partial e}{\partial e_{-1}}\right|_{\mathbb{C}}
\end{array}\right]
$$

The null block $\mathbf{0}$ prevents the block from having any long-lasting effect on the steady state stability. Under Assumption 1, the rate of growth of paid dividends is strictly positive and the deterministic eigenvalues associated with the block are always non-negative and less than unity:

$$
\begin{aligned}
& \lambda_{i}=\frac{1}{1+g}<1 \text { if and only if } g>0, \\
& \lambda_{2}^{\oplus}=0<1 \text {. }
\end{aligned}
$$

Therefore, the only eigenvalue relevant for the asymptotic dynamics is the one associated to the stochastic block o.

\section{E. Proof of Proposition 4}

Following Lemma 1, since the stochastic block of the Jacobian matrix (D.1) is $1 \times 1$ and non-negative, and thanks to the adjacent null block $\mathbf{0}$, the associated eigenvalue coincides with the geometric expected value of the element itself, according to the invariant distribution $\pi$ of the Markov process $\left\{x_{t}\right\}$. In particular, it holds

$$
\boldsymbol{\pi}=\left[\frac{\pi^{u}}{\pi^{u}+\pi^{d}}, \frac{\pi^{d}}{\pi^{u}+\pi^{d}}\right]
$$

and therefore the relevant eigenvalue reads

$$
\left.\rho^{\mathfrak{S}}\right|_{\mathbb{C}}=\mathbb{E}_{\mathcal{G}}^{\pi}\left[\left.\frac{\partial \varphi}{\partial \varphi_{-1}}\right|_{\mathbb{C}}\right]=\frac{\left(\bar{x}+g x^{u}\right)^{\frac{\pi^{u}}{\pi^{u}+\pi^{d}}}\left(\bar{x}+g x^{d}\right)^{\frac{\pi^{d}}{\pi^{u}+\pi^{d}}}}{\bar{x}(1+g)} .
$$

Inequality (4.3) guarantees that the eingevalue is inside the unit circle. Local asymptotic stability follows by applying the same argument used in Theorem 4.3 Bottazzi and Dindo (2014) that exploits Oseledets' multiplicative ergodic theorem and the local Hartman-Grobman theorem (Coayla-Teran and Ruffino, 2004, see Theorem 2.1 and 3.2, respectively). When the eigenvalue is outside the unit circle instability follows along the same lines. 


\section{F. Proof of Proposition 5}

Due to the strict concavity of the logarithm function, for the growth rate $\left.\rho^{\mathfrak{S}}\right|_{\mathbb{C}}$ it holds

$$
\begin{aligned}
& \log \left[\left(1+g \frac{x^{u}}{\bar{x}}\right)^{\frac{\pi^{u}}{\pi^{u}+\pi^{d}}}\left(1+g \frac{x^{d}}{\bar{x}}\right)^{\frac{\pi^{d}}{\pi^{u}+\pi^{d}}}\right]<\log \left[1+\frac{g}{\bar{x}}\left(\frac{\pi^{u}}{\pi^{u}+\pi^{d}} \cdot x^{u}+\frac{\pi^{d}}{\pi^{u}+\pi^{d}} \cdot x^{d}\right)\right] \\
& =\log \left[1+g \frac{\mathbb{E}\left[x_{t}\right]}{\bar{x}}\right] .
\end{aligned}
$$

It follows that if $\bar{x} \leq \mathbb{E}\left[x_{t}\right]$ then $\left.\rho^{\mathfrak{S}}\right|_{\mathbb{C}}<\left.\rho^{\mathbb{C}}\right|_{\mathbb{C}}$.

\section{G. Proof of Proposition 6}

$\left.\rho^{\mathfrak{S}}\right|_{\mathbb{C}}(\cdot)$ is a continuous and strictly decreasing function of $\bar{x}$ on the open interval $(0,1)$ as it holds

$$
\left.\frac{\partial}{\partial \bar{x}} \rho^{\mathfrak{S}}\right|_{\mathbb{C}}(\cdot)=-g \frac{\sum_{i \in\{u, d\}, j \in\{u, d\}, i \neq j} \pi^{i} x^{i}\left(\bar{x}+g x^{i}\right)^{\frac{\pi^{i}}{\pi^{i}+\pi^{j}}}\left(\bar{x}+g x^{j}\right)^{\frac{\pi^{i}+2 \pi^{j}}{\pi^{i}+\pi^{j}}}}{\bar{x}^{2}\left(\pi^{u}+\pi^{d}\right)(1+g)\left(\bar{x}+g x^{u}\right)\left(\bar{x}+g x^{d}\right)}<0 .
$$

Moreover, its limiting behaviour at the extrema of the support is characterised by

$$
\begin{aligned}
& \left.\lim _{\bar{x} \rightarrow 0^{+}} \rho^{\mathfrak{S}}\right|_{\mathbb{C}}(\cdot)=+\infty, \\
& \left.\lim _{\bar{x} \rightarrow 1^{-}} \rho^{\mathfrak{S}}\right|_{\mathbb{C}}(\cdot)=\mathbb{E}_{\mathcal{G}}^{\pi}\left[\frac{1+g x_{-1}}{1+g}\right]<1 .
\end{aligned}
$$

Applying the intermediate value theorem yields the desired result.

$$
x^{d}<x^{\prime}
$$

If $\bar{x}=x^{d}$, then condition (4.3) is violated since

$$
\left[\frac{x^{d}+g x^{u}}{x^{d}+g x^{d}}\right]^{\frac{\pi^{u}}{\pi^{u}+\pi^{d}}}>1 .
$$

Since by Proposition $\left.6 \rho^{\mathfrak{s}}\right|_{\mathbb{C}}(\cdot)$ is monotone decreasing in $\bar{x}$, it must be that $x^{\prime}>x^{d}$.

$$
x^{\prime}<x^{u}
$$


If $\bar{x}=x^{u}$, then LHS of Eq. (4.3) reads

$$
\left[\frac{x^{u}+g x^{d}}{x^{u}+g x^{u}}\right]^{\frac{\pi^{d}}{\pi^{u}+\pi^{d}}}<1 .
$$

Since by Proposition $\left.6 \rho^{\mathfrak{S}}\right|_{\mathbb{C}}(\cdot)$ is monotone decreasing in $\bar{x}$, by continuity $\exists \varepsilon>0$ such that $\left.\rho^{\mathfrak{S}}\right|_{\mathbb{C}}\left(x^{u}-\varepsilon\right)<1$. Therefore, it must be that $x^{\prime}<x^{u}$.

\section{H. Proof of Proposition 7}

Consider the stochastic Jacobian matrix $\mathcal{J}^{\mathfrak{S}}$ of system (2.15) computed at the steady state S. It is straightforward to check that $\mathcal{J}^{\mathfrak{S}}$ is structurally analogous to $\mathcal{J}^{\mathfrak{C}}$, and therefore it is possible to apply Lemma 1 . Since the stochastic block of $\mathcal{J}^{\mathfrak{S}}$ is $1 \times 1$ and nonnegative, and thanks to the adjacent null block 0 , the associated eigenvalue coincides with the geometric expected value of the element itself, according to the invariant distribution $\tilde{\pi}$ of the stochastic limit 4-cycle derived in Corollary 1, i.e.

$$
\left.\rho^{\mathfrak{C}}\right|_{\mathfrak{S}}=\mathbb{E}_{\mathcal{G}}^{\tilde{\pi}}\left[\left.\frac{\partial \varphi}{\partial \varphi_{-1}}\right|_{\mathfrak{S}}\right]
$$

Inequality (4.5) guarantees that the usual unit circle condition is satisfied. Local asymptotic stability and instability follow as for the proof of Proposition 4 in Appendix E.

\section{Proof of Proposition 8}

Start by decomposing Eq. (4.5) in its multiplicative components. Let us define $\left.\rho_{i j}^{\mathbb{C}}\right|_{\mathfrak{S}}$ as the value of $\left.\frac{\partial \varphi}{\partial \varphi_{-1}}\right|_{\mathfrak{S}}$ when $x_{-1}=x^{i}$ and $x=x^{j}$, with $x^{i}, x^{j} \in\left\{x^{u}, x^{d}\right\}$. Note that we can safely get rid of the probability exponent since it plays no role in the current analysis. It is straightforward to check that

$$
\begin{aligned}
& \left.\rho_{u u}^{\mathbb{C}}\right|_{\mathfrak{S}}<1 \quad \Longleftrightarrow \bar{x}<x^{u}, \\
& \left.\rho_{u d}^{\mathbb{C}}\right|_{\mathfrak{S}}<1 \quad \Longleftrightarrow \bar{x}>x^{u} \quad \underline{\vee} \quad g>\frac{x^{u}-x^{d}}{1-x^{u}}, \\
& \left.\rho_{d u}^{\mathbb{C}}\right|_{\mathfrak{S}}<1 \quad \Longleftrightarrow \bar{x}<x^{d}, \\
& \left.\rho_{d d}^{\mathbb{C}}\right|_{\mathfrak{S}}<1 \quad \Longleftrightarrow \bar{x}<x^{d},
\end{aligned}
$$


where the symbol $\underline{\vee}$ stands for the logical exclusive disjunction operator. Conditions (4.6) and (4.7) follow from the joint satisfaction (dissatisfaction, respectively) of the four statements above.

\section{J. Hint of Conjecture 1}

$\left.\rho^{\mathfrak{C}}\right|_{\mathfrak{S}}(\cdot)$ is a continuous and differentiable function of $\bar{x}$ over the open interval $(0,1)$. At the extrema of the support it holds

$$
\begin{aligned}
& \left.\lim _{\bar{x} \rightarrow 0^{+}} \rho^{\mathbb{C}}\right|_{\mathfrak{S}}(\cdot)=\frac{1}{1+g}<1 \\
& \left.\lim _{\bar{x} \rightarrow 1^{-}} \rho^{\mathfrak{C}}\right|_{\mathfrak{S}}(\cdot)=\frac{1}{1+g} \cdot \mathbb{E}_{\mathcal{G}}^{\tilde{\tilde{G}}}\left[1+\frac{g}{x_{-1}}\right]>1 .
\end{aligned}
$$

Moreover, it is possible to numerically show that $\left.\rho^{\mathbb{C}}\right|_{\mathfrak{S}}(\cdot)$ exhibit no inflection points over $\bar{x} \in(0,1)$. We look for the roots of $\left.\frac{\partial^{2}}{\partial \bar{x}^{2}} \rho^{\mathbb{C}}\right|_{\mathfrak{S}}(\cdot)=0$ using the Anderson-Björck method $\forall x^{u}, x^{d}, \pi^{u}, \pi^{d} \in\{0.01,0.02, \ldots, 0.99\}$ such that $x^{u}>x^{d}, \forall g \in 10^{k}, k \in\{-4,-3, \ldots,+1\}$. We then check that none of them belong to the interval $(0,1)$, finding no exceptions. Applying the intermediate value theorem yields the desired result.

\section{K. Proof of Proposition 9}

Start by decomposing Eq. (4.5) in its multiplicative components. Let us define $\left.\rho_{i j}^{\mathfrak{C}}\right|_{\mathfrak{S}}$ as the value of $\left.\frac{\partial \varphi}{\partial \varphi_{-1}}\right|_{\mathfrak{S}}$ when $x_{-1}=x^{i}$ and $x=x^{j}$, with $x^{i}, x^{j} \in\left\{x^{u}, x^{d}\right\}$. It is straightforward to check that if $\bar{x}=x^{d}$ then $\left.\rho_{d u}^{\mathbb{C}}\right|_{\mathfrak{S}}\left(x^{d}\right)=\left.\rho_{d d}^{\mathbb{C}}\right|_{\mathfrak{S}}\left(x^{d}\right)=1$ and $\left.\rho^{\mathbb{C}}\right|_{\mathfrak{S}}$ reduces to

$$
\left.\rho^{\mathbb{C}}\right|_{\mathfrak{S}}\left(x^{d}\right)=\left.\left.\rho_{u \mathfrak{u}}^{\mathbb{C}}\right|_{\mathfrak{S}}\left(x^{d}\right)^{\frac{\pi^{u}\left(1-\pi^{d}\right)}{\pi^{u}+\pi^{d}}} \cdot \rho_{u d}^{\mathbb{C}}\right|_{\mathfrak{S}}\left(x^{d}\right)^{\frac{\pi^{u} \pi^{d}}{\pi^{u}+\pi^{d}}} .
$$

From Proposition 8 it follows that

$$
\begin{aligned}
& \left.\rho_{u u}^{\mathbb{C}}\right|_{\mathfrak{S}}\left(x^{d}\right)<1, \\
& \left.\rho_{u d}^{\mathfrak{C}}\right|_{\mathfrak{S}}\left(x^{d}\right) \gtreqless 1 \quad \Longleftrightarrow g \lesseqgtr \frac{x^{u}-x^{d}}{1-x^{u}} .
\end{aligned}
$$

Moreover,

$$
\begin{aligned}
& \left.\lim _{g \rightarrow 0^{+}} \rho_{u u}^{\mathfrak{c}}\right|_{\mathfrak{S}}\left(x^{d}\right)=1, \\
& \left.\lim _{g \rightarrow 0^{+}} \rho_{u d}^{\mathfrak{c}}\right|_{\mathfrak{S}}\left(x^{d}\right)=\frac{x^{u}+x^{d}\left(2 x^{u}-x^{d}\right)}{x^{u}\left(1-x^{u}\right)}>1 .
\end{aligned}
$$


Therefore, there exists a $\hat{g}>0$ such that $\forall g<\hat{g}$ condition (4.5) is strictly violated, i.e. $\left.\rho^{\mathbb{C}}\right|_{\mathfrak{S}}\left(x^{d}\right)>1$. Since from Conjecture 1 the solution to the problem $\left.\rho^{\mathbb{C}}\right|_{\mathfrak{S}}\left(x^{d}\right)=1$ is unique, then $\forall g<\hat{g}$ it must be that $x^{\prime \prime}<x^{d}$. Finally, Proposition 6 implies that in such cases the following relations hold:

$$
x^{\prime \prime}<x^{d}<x^{\prime}
$$

\section{Hint of Conjecture 2}

The unique solution $x^{\prime}$ of $\left.\rho^{\mathfrak{S}}\right|_{\mathbb{C}}(\bar{x})=1$ is computed using the Anderson-Björck method and then the condition $\left.\rho^{\mathbb{C}}\right|_{\mathfrak{S}}\left(x^{\prime}\right)>1$ is tested $\forall x^{u}, x^{d}, \pi^{u}, \pi^{d} \in\{0.01,0.02, \ldots, 0.99\}$ such that $x^{u}>x^{d}$, and $\forall g=\hat{g} \cdot 10^{k}, k \in \mathbb{N}_{+}$under the restriction $g \leq 10$. No exception is found. 